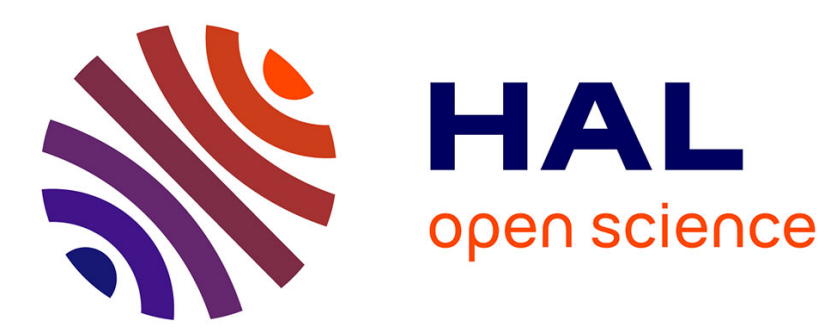

\title{
Preferences for multi-attributed alternatives: Traces, Dominance, and Numerical Representations
}

\author{
Denis Bouyssou, Marc Pirlot
}

\section{To cite this version:}

Denis Bouyssou, Marc Pirlot. Preferences for multi-attributed alternatives: Traces, Dominance, and Numerical Representations. Journal of Mathematical Psychology, 2004, 48 (3), pp.167-185. 10.1016/j.jmp.2004.02.005 . hal-00004104

\section{HAL Id: hal-00004104 https://hal.science/hal-00004104}

Submitted on 31 Jan 2005

HAL is a multi-disciplinary open access archive for the deposit and dissemination of scientific research documents, whether they are published or not. The documents may come from teaching and research institutions in France or abroad, or from public or private research centers.
L'archive ouverte pluridisciplinaire HAL, est destinée au dépôt et à la diffusion de documents scientifiques de niveau recherche, publiés ou non, émanant des établissements d'enseignement et de recherche français ou étrangers, des laboratoires publics ou privés. 


\title{
Preferences for multi-attributed alternatives: Traces, Dominance, and Numerical Representations ${ }^{1}$
}

\author{
D. Bouyssou ${ }^{2}$ \\ M. Pirlot ${ }^{3}$ \\ CNRS - LAMSADE \\ Faculté Polytechnique de Mons
}

Revised 13 November 2002

\begin{abstract}
${ }^{1}$ Part of this work was accomplished while Denis Bouyssou was visiting the Service de Mathématiques de la Gestion at the Université Libre de Bruxelles (Brussels, Belgium). He gratefully acknowledges the warm hospitality of the Service de Mathématique de la Gestion as well as the support of the Belgian Fonds National de la Recherche Scientifique and the Brussels-Capital Region through a "Research in Brussels" action grant.The other part of this work was accomplished while Marc Pirlot was visiting LIP6-Université Pierre et Marie Curie and LAMSADEUniversité Paris Dauphine (Paris, France) thanks to visiting positions in these two institutions and a grant from the Fonds National de la Recherche Scientifique. He gratefully acknowledges this support.

${ }^{2}$ LAMSADE, Université Paris Dauphine, Place du Maréchal de Lattre de Tassigny, F-75775 Paris Cedex 16, France, tel: +33 1440548 98, fax: +3314405 40 91, e-mail: bouyssou@lamsade.dauphine.fr, Corresponding author.

${ }^{3}$ Faculté Polytechnique de Mons, 9, rue de Houdain, B-7000 Mons, Belgium, tel: +3265374682 , fax: +3265374689 , e-mail: pirlot@mathro.fpms.ac.be.
\end{abstract}




\begin{abstract}
This paper analyzes conjoint measurement models allowing for intransitive and/or incomplete preferences. This analysis is based on the study of marginal traces induced on coordinates by the preference relation and uses conditions guaranteeing that these marginal traces are complete.

Within the framework of these models, we propose a simple axiomatic characterization of preference relations compatible with the notion of dominance. We show that all such relations have a nontrivial numerical representation.

Our results allow us to establish useful connections between two lines of thought in the area of decision analysis with multiple attributes that have largely remained unrelated: the one based on conjoint measurement and the one emphasizing the idea of dominance.
\end{abstract}

Keywords: Conjoint measurement, Nontransitive preferences, Decision analysis with multiple attributes, Dominance, Traces. 


\section{Motivation and outline}

Two distinct traditions underlie most of the work done in the area of decision analysis with multiple attributes. The conjoint measurement tradition has deep roots both in Mathematical Psychology and Decision Theory (see Debreu, 1960; Krantz, Luce, Suppes, \& Tversky, 1971; Luce \& Tukey, 1964; Roberts, 1979; Scott, 1964; Scott \& Suppes, 1958; Wakker, 1989). Starting with a binary relation $\succsim$ defined on a product set $X=X_{1} \times X_{2} \times \cdots \times X_{n}$, its aim is to find conditions under which it is possible to build a convenient numerical representation of $\succsim$. The model that has been most studied in this framework is the additive utility model:

$$
x \succsim y \Leftrightarrow \sum_{i=1}^{n} u_{i}\left(x_{i}\right) \geq \sum_{i=1}^{n} u_{i}\left(y_{i}\right),
$$

where $u_{i}$ is a real-valued function on $X_{i}$ and it is understood that $x=$ $\left(x_{1}, x_{2}, \ldots, x_{n}\right)$ and $y=\left(y_{1}, y_{2}, \ldots, y_{n}\right)$.

Besides their theoretical interest and the fact that they exhibit conditions likely to be subjected to empirical tests, many conjoint measurement results are constructive in nature and, therefore, give hints on how to devise assessment procedures of utility functions and, thus, preferences. Indeed, the framework of conjoint measurement has been adopted in many important works in decision analysis (see French, 1993; Keeney \& Raiffa, 1976; Winterfeldt \& Edwards, 1986) giving rise to many specialized assessment techniques (see Belton \& Stewart, 2001; Bouyssou et al., 2000; Keeney \& Raiffa, 1976)) that have often been applied in real-world settings. Note that most developments in conjoint measurement require that $\succsim$ is very well-behaved being, in particular, complete and transitive.

A more pragmatic tradition starts with alternatives evaluated along several attributes. Along each attribute, alternatives are supposed to be compared using a well-behaved preference relation. The central problem is then to build a preference relation between alternatives taking all attributes into account, i.e. a global preference relation, based on the preference relations on each attribute and "inter-attribute" information such as weights or trade-offs (Pomerol \& Barba-Romero, 2000; Roy, 1996; Roy \& Bouyssou, 1993; Steuer, 1986; Vincke, 1992). The notion of dominance plays a crucial role here. An alternative $x$ is said to dominate an alternative $y$ if $x$ is judged "at least as good as" as $y$ on all attributes. Suppose that $z$ dominates $x$ and that $y$ dominates $w$. If we have reasons to believe that " $x$ is at least as good as $y$ " and if we want the global preference relation to be compatible with dominance then we should judge $z$ at least as good as $w$. When a global preference relation 
is compatible with dominance, it makes sense to limit the search for "good" alternatives in the set of efficient alternatives, i.e. alternatives that are undominated. Most techniques related to the pragmatic tradition heavily rely on the notion of dominance (see Pomerol \& Barba-Romero, 2000; Vincke, 1992). When the set of alternatives is "large", e.g. in the case of multiobjective optimization, many methods have been devoted to the identification of efficient alternatives (see Steuer, 1986).

These two lines of thought seem to coexist since the beginning of decision analysis with multiple attributes, in the late '60s (see Raiffa, 1968; Roy, 1971). Both have generated important theoretical and practical achievements. Their setting differ significantly. The conjoint measurement tradition starts with a well-behaved preference relation taking all attributes into account. The pragmatic one starts with a well-behaved preference relation defined on each attribute and derives a global preference relation using the notion of dominance and inter-attribute information. The principles used in order to build the global preference relation do not always guarantee that this relation will be transitive or complete, e.g. if a qualified weighted majority of attributes is used (see Roy, 1991; Vincke, 1992). The sad consequence is that these two traditions have largely remained unrelated. Indeed, the idea of dominance receives little attention in most books related to the conjoint measurement tradition (French, 1993; Keeney \& Raiffa, 1976). Conversely, in many books related to the pragmatic tradition, conjoint measurement approaches, are either omitted or treated apart from anything else (Goicoechea, Hansen, \& Duckstein, 1982; Steuer, 1986; Zeleny, 1982).

This paper is an attempt to establish connections between these two traditions. In order to do so, we adopt a classical conjoint measurement setting, while not requiring transitivity or completeness. We provide a simple axiomatic characterization of preference relations compatible with dominance and show that all such relations admit a nontrivial numerical representation. This extends the traditional scope of conjoint measurement to include binary relations that are not "well-behaved". Furthermore this shows that many techniques developed in the pragmatic tradition can usefully be analyzed in a conjoint measurement framework.

Technically, we pursue a line of investigation started in a series of earlier papers (Bouyssou, Pirlot, \& Vincke, 1997; Bouyssou \& Pirlot, 1999, 2002a), and anticipated in Goldstein (1991), consisting in analyzing conjoint measurement models that involve neither transitivity nor additivity. The key tool for the analysis of such preference relations is the consideration of various kinds of traces on coordinates induced by the original relation.

This paper is organized as follows. Section 2 presents some background material: we introduce our vocabulary concerning binary relations and recall 
some well-known facts on traces. Section 3 studies binary relations defined on product sets and introduces the notion of marginal trace. Using conditions implying that marginal traces are complete, section 4 offers a simple characterization of preference relations compatible with the notion of dominance. Section 5 shows that all such preference relations admit several kinds of nontrivial numerical representations whether or not they are transitive or complete. Section 6 discusses our results and presents directions for future research. Examples and technical details are relegated in appendix.

\section{Background: Binary relations and traces}

\subsection{Binary relations}

A binary relation $\succsim$ on a set $A$ is a subset of $A \times A$. We write $a \succsim b$ instead of $(a, b) \in \succsim$. A binary relation $\succsim$ on $A$ is said to be:

- reflexive if $[a \succsim a]$,

- complete if $[a \succsim b$ or $b \succsim a]$,

- symmetric if $[a \succsim b] \Rightarrow[b \succsim a]$,

- asymmetric if $[a \succsim b] \Rightarrow[N o t[b \succsim a]]$,

- transitive if $[a \succsim b$ and $b \succsim c] \Rightarrow[a \succsim c]$,

- Ferrers if

$$
\left.\begin{array}{c}
a \succsim b \\
\text { and } \\
c \succsim d
\end{array}\right\} \Rightarrow\left\{\begin{array}{c}
a \succsim d \\
\text { or } \\
c \succsim b,
\end{array}\right.
$$

- semi-transitive if

$$
\left.\begin{array}{c}
a \succsim b \\
\text { and } \\
b \succsim c
\end{array}\right\} \Rightarrow\left\{\begin{array}{c}
a \succsim d \\
\text { or } \\
d \succsim c
\end{array}\right.
$$

for all $a, b, c, d \in A$.

The asymmetric (resp. symmetric) part of $\succsim$ is the binary relation $\succ$ (resp. $\sim$ ) on $A$ defined letting, for all $a, b \in A, a \succ b \Leftrightarrow[a \succsim b$ and $\operatorname{Not}(b \succsim$ $a)]$ (resp. $a \sim b \Leftrightarrow[a \succsim b$ and $b \succsim a]$ ). A similar convention will hold when $\succsim$ is subscripted and/or superscripted.

A weak order (resp. an equivalence relation) is a complete and transitive (resp. reflexive, symmetric and transitive) binary relation. A complete order 
is a weak order the symmetric part of which is limited to loops. An interval order is a complete and Ferrers binary relation; a semi-order is a semitransitive interval order. If $\succsim$ is an equivalence on $A, A / \succsim$ will denote the set of equivalence classes of $\succsim$ on $A$.

\subsection{Traces of binary relations}

The idea that any binary relation generates various reflexive and transitive binary relations called traces dates back at least to Luce (1956) (in order to distinguish them from traces on coordinates when studying product sets, we will later designate these traces as global traces). The use of traces have proved especially useful in the study of preference structures tolerating imperfect discrimination such as semi-orders, interval orders or valued preference relations (Doignon, Monjardet, Roubens, \& Vincke, 1988; Fishburn, 1985; Pirlot \& Vincke, 1997) and in Social Choice Theory under the name of "covering relations" (Laslier, 1997). These relations will also prove important in what follows.

\section{Definition 1 (Global traces)}

Let $\succsim$ be a binary relation on a set $A$. We associate to $\succsim$ three binary relations, called traces, letting, for all $a, b \in A$ :

Left Trace $a \succsim^{+} b \Leftrightarrow[b \succsim c \Rightarrow a \succsim c]$,

Right Trace $a \succsim^{-} b \Leftrightarrow[c \succsim a \Rightarrow c \succsim b]$,

Trace $a \succsim^{ \pm} b \Leftrightarrow\left[a \succsim^{+} b\right.$ and $\left.a \succsim^{-} b\right]$.

Following our conventions, $\sim^{+}$and $\succ^{+}$will denote the symmetric and asymmetric parts of $\succsim^{+}$, the same being true for $\succsim^{-}$and $\succsim^{ \pm}$. Useful connections between $\succsim$ and its traces are summarized below for the ease of future reference. All of them are straightforward consequences of the preceding definition.

\section{Proposition 1 (Properties of global traces)}

1. $\sim^{+}, \sim^{-}$and $\sim^{ \pm}$are equivalence relations (reflexive, symmetric and transitive).

2. $\succsim^{+}, \succsim^{-}$and $\succsim^{ \pm}$are reflexive and transitive binary relations.

3. For all $a, b, c, d \in A$ :

$$
\begin{aligned}
& {\left[a \succsim b, b \succsim^{-} c\right] \Rightarrow a \succsim c,} \\
& {\left[a \succsim b, c \succsim^{+} a\right] \Rightarrow c \succsim b,}
\end{aligned}
$$




$$
\begin{aligned}
& {\left[d \succsim^{ \pm} a, b \succsim^{ \pm} c\right] \Rightarrow\left\{\begin{array}{l}
a \succsim b \Rightarrow d \succsim c, \\
a n d \\
a \succ b \Rightarrow d \succ c
\end{array}\right.} \\
& {\left[a \sim^{ \pm} c, b \sim^{ \pm} d\right] \Rightarrow\left\{\begin{array}{l}
a \succsim b \Leftrightarrow c \succsim d, \\
a n d \\
a \succ b \Leftrightarrow c \succ d .
\end{array}\right.}
\end{aligned}
$$

4. $\succsim^{ \pm}=\succsim \Leftrightarrow \succsim$ is reflexive and transitive.

5. $\left[\succsim^{ \pm}=\succsim\right.$ and $\succsim^{ \pm}$is complete $] \Leftrightarrow \succsim$ is a weak order.

The following proposition summarizes a number of well-known facts about traces (see Fishburn, 1985; Monjardet, 1978; Pirlot \& Vincke, 1997; Roubens \& Vincke, 1985).

\section{Proposition 2 (Completeness of global traces)}

1. $\succsim^{+}$is complete $\Leftrightarrow \succsim^{-}$is complete $\Leftrightarrow \succsim$ is Ferrers.

2. $\succsim^{ \pm}$is complete $\Leftrightarrow \succsim$ is Ferrers and semi-transitive.

For a detailed analysis of the role of traces in various domains of preference modelling we refer to Aleskerov and Monjardet (2002), Doignon et al. (1988), Laslier (1997), Monjardet (1978), Pirlot and Vincke (1997), Roubens and Vincke (1985).

\section{Binary relations on product sets}

We consider now a set $X=\prod_{i=1}^{n} X_{i}$ with $n \geq 2$. Elements $x, y, z, \ldots$ of $X$ will be interpreted as alternatives evaluated on a set $N=\{1,2, \ldots, n\}$ of attributes. A typical binary relation on $X$ is still denoted as $\succsim$. It is useful to interpret $\succsim$ as an "at least as good as" preference relation between multiattributed alternatives with $\sim$ interpreted as indifference and $\succ$ as strict preference.

For any non empty subset $J$ of the set of attributes $N$, we denote by $X_{J}$ (resp. $\left.X_{-J}\right)$ the set $\prod_{i \in J} X_{i}$ (resp. $\left.\prod_{i \notin J} X_{i}\right)$. With customary abuse of notation, $\left(x_{J}, y_{-J}\right)$ will denote the element $w \in X$ such that $w_{i}=x_{i}$ if $i \in J$ and $w_{i}=y_{i}$ otherwise. When $J=\{i\}$ we shall simply write $X_{-i}$ and $\left(x_{i}, y_{-i}\right)$.

We say that $\succsim$ is marginally complete for $i \in N$ if $\left(x_{i}, a_{-i}\right) \succsim\left(y_{i}, a_{-i}\right)$ or $\left(y_{i}, a_{-i}\right) \succsim\left(x_{i}, a_{-i}\right)$, for all $x_{i}, y_{i} \in X_{i}$ and all $a_{-i} \in X_{-i}$, i.e. if no incomparability occurs when comparing alternatives differing only on attribute $i \in N$. 


\subsection{Independence and marginal preferences}

In conjoint measurement, one starts with a preference relation $\succsim$ on $X$. It is then of vital importance to investigate how this information makes it possible to define preference relations on attributes or subsets of attributes.

Let $J \subseteq N$ be a nonempty set of attributes. We define the marginal relation $\succsim_{J}$ induced on $X_{J}$ by $\succsim$ letting, for all $x_{J}, y_{J} \in X_{J}$ :

$$
x_{J} \succsim_{J} y_{J} \Leftrightarrow\left(x_{J}, z_{-J}\right) \succsim\left(y_{J}, z_{-J}\right) \text {, for all } z_{-J} \in X_{-J},
$$

with asymmetric (resp. symmetric) part $\succ_{J}\left(\right.$ resp. $\left.\sim_{J}\right)$. Note that if $\succsim$ is reflexive (resp. transitive), the same will be true for $\succsim_{J}$. This is clearly not true for completeness however.

We define two other binary relations $R_{\widetilde{J}}^{\succsim}$ and $R_{J}^{\succ}$ induced by $\succsim$ on $X_{J}$, letting for all $x_{J}, y_{J} \in X_{J}$,

$$
x_{J} R_{\widetilde{J}}^{\succsim} y_{J} \Leftrightarrow\left(x_{J}, z_{-J}\right) \succsim\left(y_{J}, z_{-J}\right) \text {, for some } z_{-J} \in X_{-J},
$$

and

$$
x_{J} R_{J}^{\succ} y_{J} \Leftrightarrow\left(x_{J}, z_{-J}\right) \succ\left(y_{J}, z_{-J}\right) \text {, for some } z_{-J} \in X_{-J} .
$$

\section{Definition 2 (Independence and separability)}

Consider a binary relation $\succsim$ on a set $X=\prod_{i=1}^{n} X_{i}$ and let $J \subseteq N$ be a nonempty subset of attributes. We say that $\succsim$ is:

1. independent for $J$ if $R_{\widetilde{J}}^{\succsim} \subseteq \succsim_{J}$,

2. separable for $J$ if $R_{J}^{\succ}$ is asymmetric.

If $\succsim$ is independent (resp. separable) for all non empty subsets of $N$, we say that $\succsim$ is independent (resp. separable). If $\succsim$ is independent (resp. separable) for all subsets containing a single attribute, we say that $\succsim$ is weakly independent (resp. weakly separable).

Independence is a classical notion in conjoint measurement. It states that common evaluations on some attributes do not influence preference. Whereas independence implies weak independence, it is well-know that the converse is not true (see Wakker, 1989).

Independence implies separability but not vice versa. Separability is a weakening of independence that can be motivated considering aggregation models based on "max" or "min". It forbids strict reversals of preference when varying common evaluations on some attribute. In special contexts, it has already been considered in Blackorby, Primont, and Russell (1978), Färe 
and Primont (1981), Mak (1984), Segal and Sobel (2001). It is easy to see that weak separability does not entail separability.

Let us observe that when $\succsim$ is complete and independent for $i \in N$ then $\succsim_{i}$ is clearly complete. It is not difficult to see that $\succsim_{i}$ is complete if and only if $\succsim$ is marginally complete and weakly separable for $i \in N$.

\subsection{Marginal traces}

The definitions and results from section 2.2 clearly apply here. Hence the binary relation $\succsim$ on $X=\prod_{i=1}^{n} X_{i}$ has a left trace (resp. right trace and trace $) \succsim^{+}$(resp. $\succsim^{-}$and $\succsim^{ \pm}$) that is reflexive and transitive.

Consider an attribute $i \in N$. Sticking to the notation introduced above, $\succsim_{i}^{+}$(resp. $\succsim_{i}^{-}$and $\succsim_{i}^{ \pm}$) will denote the marginal preference relation induced on $X_{i}$ by $\succsim^{+}$(resp. $\succsim^{-}$and $\succsim^{ \pm}$), i.e.

$$
\begin{aligned}
& x_{i} \succsim_{i}^{+} y_{i} \Leftrightarrow\left[\left(x_{i}, z_{-i}\right) \succsim^{+}\left(y_{i}, z_{-i}\right), \text { for all } z_{-i} \in X_{-i}\right], \\
& x_{i} \succsim_{i}^{-} y_{i} \Leftrightarrow\left[\left(x_{i}, z_{-i}\right) \succsim^{-}\left(y_{i}, z_{-i}\right), \text { for all } z_{-i} \in X_{-i}\right], \\
& x_{i} \succsim_{i}^{ \pm} y_{i} \Leftrightarrow\left[\left(x_{i}, z_{-i}\right) \succsim^{ \pm}\left(y_{i}, z_{-i}\right), \text { for all } z_{-i} \in X_{-i}\right] .
\end{aligned}
$$

Since, by construction, $\succsim^{+}, \succsim^{-}$and $\succsim^{ \pm}$are reflexive and transitive, the same is true for $\succsim_{i}^{+}, \succsim_{i}^{-}$and $\succsim_{i}^{ \pm}$. From proposition 2 , we know that $\succsim^{\prime} \succsim^{ \pm}$if and only if $\succsim$ is reflexive and transitive. When this is the case, we clearly have $\succsim_{i}=\succsim_{i}^{ \pm}$, for all $i \in X$. As shown in the following lemma, $\succsim_{i}^{+}$(resp. $\succsim_{i}^{-}$and $\left.\succsim_{i}^{ \pm}\right)$, the marginal relation induced on $i \in N$ by the global trace $\succsim^{+}$(resp. $\succsim_{i}^{-}$and $\succsim_{i}^{ \pm}$) can also be usefully interpreted as a marginal trace on attribute $i \in N$.

\section{Lemma 1 (Marginal relations induced by global traces)}

For all $i \in N$, all $x_{i}, y_{i} \in X_{i}$, all $a_{-i} \in X_{-i}$ and all $z \in X$ :

$$
\begin{aligned}
& \text { 1. } x_{i} \succsim_{i}^{+} y_{i} \Leftrightarrow\left[\left(y_{i}, a_{-i}\right) \succsim z \Rightarrow\left(x_{i}, a_{-i}\right) \succsim z\right], \\
& \text { 2. } x_{i} \succsim_{i}^{-} y_{i} \Leftrightarrow\left[z \succsim\left(x_{i}, a_{-i}\right) \Rightarrow z \succsim\left(y_{i}, a_{-i}\right)\right], \\
& \text { 3. } x_{i} \succsim_{i}^{ \pm} y_{i} \Leftrightarrow\left\{\begin{array}{l}
\left(y_{i}, a_{-i}\right) \succsim z \Rightarrow\left(x_{i}, a_{-i}\right) \succsim z, \\
\text { and } \\
z \succsim\left(x_{i}, a_{-i}\right) \Rightarrow z \succsim\left(y_{i}, a_{-i}\right) .
\end{array}\right.
\end{aligned}
$$

\section{PROOF}

We give the proof of part 1, the proof of the other parts being similar. By definition we have: $x_{i} \succsim_{i}^{+} y_{i} \Leftrightarrow\left[\left(x_{i}, a_{-i}\right) \succsim^{+}\left(y_{i}, a_{-i}\right)\right.$, for all $\left.a_{-i} \in X_{-i}\right] \Leftrightarrow$ $\left[\left(y_{i}, a_{-i}\right) \succsim z \Rightarrow\left(x_{i}, a_{-i}\right) \succsim z\right.$, for all $a_{-i} \in X_{-i}$ and all $\left.z \in X\right]$. 
As before, the symmetric and asymmetric parts of $\succsim_{i}^{+}$are respectively denoted $\sim_{i}^{+}$and $\succ_{i}^{+}$, the same convention applying to $\succsim_{i}^{-}$and $\succsim_{i}^{ \pm}$. Although it is clearly possible to define marginal traces on subsets of attributes more general than singletons, we do not envisage this possibility here.

As in proposition 1, there are many interesting connections between marginal traces and $\succsim$. We list some of them in the following lemma, for the ease of future reference, omitting its obvious proof.

\section{Lemma 2 (Properties of marginal traces)}

For all $i \in N$ and $x, y, z, w \in X$ :

$$
\begin{gathered}
{\left[x \succsim y, z_{i} \succsim_{i}^{+} x_{i}\right] \Rightarrow\left(z_{i}, x_{-i}\right) \succsim y,} \\
{\left[x \succsim y, y_{i} \succsim_{i}^{-} w_{i}\right] \Rightarrow x \succsim\left(w_{i}, y_{-i}\right),} \\
{\left[z_{i} \succsim_{i}^{ \pm} x_{i}, y_{i} \succsim_{i}^{ \pm} w_{i}\right] \Rightarrow\left\{\begin{array}{l}
x \succsim y \Rightarrow\left(z_{i}, x_{-i}\right) \succsim\left(w_{i}, y_{-i}\right), \\
a n d \\
x \succ y \Rightarrow\left(z_{i}, x_{-i}\right) \succ\left(w_{i}, y_{-i}\right),
\end{array}\right.} \\
{\left[x_{i} \sim_{i}^{ \pm} z_{i}, y_{i} \sim_{i}^{ \pm} w_{i} \text { for all } i \in N\right] \Rightarrow\left\{\begin{array}{l}
x \succsim y \Leftrightarrow z \succsim w, \\
a n d \\
x \succ y \Leftrightarrow z \succ w .
\end{array}\right.}
\end{gathered}
$$

It is clear that the marginal traces $\succsim_{i}^{+}, \succsim_{i}^{-}$and $\succsim_{i}^{ \pm}$need not be complete. Interesting consequences will arise when this is the case. This is explored in what follows.

\subsection{Complete marginal traces}

As was the case with the Ferrers and semi-transitivity conditions when studying global traces, we envisage here conditions that will guarantee that marginal traces are complete and, hence, weak orders. As with interval orders and semi-orders, these conditions will prove useful to analyze the underlying structures and to build numerical representations.

Definition 3 (Conditions $A C 1, A C 2$ and $A C 3$ )

We say that $\succsim$ satisfies:

$A C 1_{i}$ if

$$
\left.\begin{array}{c}
x \succsim y \\
\text { and } \\
z \succsim w
\end{array}\right\} \Rightarrow\left\{\begin{array}{c}
\left(z_{i}, x_{-i}\right) \succsim y, \\
o r \\
\left(x_{i}, z_{-i}\right) \succsim w
\end{array}\right.
$$

$A C 2_{i}$ if

$$
\left.\begin{array}{c}
x \succsim y \\
\text { and } \\
z \succsim w
\end{array}\right\} \Rightarrow\left\{\begin{array}{c}
x \succsim\left(w_{i}, y_{-i}\right), \\
o r \\
z \succsim\left(y_{i}, w_{-i}\right),
\end{array}\right.
$$


$A C 3_{i}$ if

$$
\left.\begin{array}{r}
z \succsim\left(x_{i}, a_{-i}\right) \\
\text { and } \\
\left(x_{i}, b_{-i}\right) \succsim y
\end{array}\right\} \Rightarrow\left\{\begin{array}{l}
z \succsim\left(w_{i}, a_{-i}\right) \\
\text { or } \\
\left(w_{i}, b_{-i}\right) \succsim y
\end{array}\right.
$$

for all $x, y, z, w \in X$, all $a_{-i}, b_{-i} \in X_{-i}$ and all $x_{i}, w_{i} \in X_{i}$.

We say that $\succsim$ satisfies $A C 1$ (resp. AC2, AC3) if it satisfies $A C 1_{i}$ (resp. $A C 2_{i}, A C 3_{i}$ ) for all $i \in N$.

These three conditions are transparent variations on the theme of the Ferrers $(A C 1$ and $A C 2)$ and semi-transitivity $(A C 3)$ conditions that are made possible by the product structure of $X$. The rationale for the name " $A C$ " is that these conditions are "intrA-attibute Cancellation" conditions.

Condition $A C 1_{i}$ suggests that the elements of $X_{i}$ (instead of the elements of $X$ had the original Ferrers condition been invoked) can be linearly ordered considering "upward dominance": if $x_{i}$ "upward dominates" $z_{i}$ then $\left(z_{i}, c_{-i}\right) \succsim w$ entails $\left(x_{i}, c_{-i}\right) \succsim w$. Condition $A C 2_{i}$ has a similar interpretation considering now "downward dominance". Condition $A C 3_{i}$ ensures that the linear arrangements of the elements of $X_{i}$ obtained considering upward and downward dominance are not incompatible.

Conditions $A C 1, A C 2$ and $A C 3$ were introduced in Bouyssou et al. (1997) and Bouyssou and Pirlot (1999) and later used in Greco, Matarazzo, and Słowiński (2002). The strong links between $A C 1, A C 2, A C 3$ and marginal traces are noted in the following:

\section{Lemma 3 (Completeness of marginal traces)}

We have:

1. $\succsim_{i}^{+}$is complete iff $A C 1_{i}$ holds,

2. $\succsim_{i}^{-}$is complete iff $A C 2_{i}$ holds,

3. $\left[\operatorname{Not}\left[x_{i} \succsim_{i}^{+} y_{i}\right] \Rightarrow y_{i} \succsim_{i}^{-} x_{i}\right]$ iff $\left[\operatorname{Not}\left[x_{i} \succsim_{i}^{-} y_{i}\right] \Rightarrow y_{i} \succsim_{i}^{+} x_{i}\right]$ iff $A C 3_{i}$ holds,

4. $\succsim_{i}^{ \pm}$is complete iff $A C 1_{i}, A C 2_{i}$ and $A C 3_{i}$ hold,

5. In the class of complete binary relations on $X, A C 1_{i}, A C 2_{i}$ and $A C 3_{i}$ are independent conditions.

\section{PROOF}

Part 1 is proved observing that the negation of $A C 1_{i}$ is equivalent to the negation of the completeness of $\succsim_{i}^{+}$. The proof of part 2 is similar. Part 3 is proved observing that the negation of $A C 3_{i}$ is equivalent to $\operatorname{Not}\left[y_{i} \succsim_{i}^{+} x_{i}\right]$ 
and $\operatorname{Not}\left[x_{i} \succsim_{i}^{-} y_{i}\right]$ for some $x_{i}, y_{i} \in X_{i}$. Part 4 immediately results from parts 1,2 and 3 .

Part 5: see examples 1, 2 and 3 in appendix A.

Comparing lemma 3 with proposition 2 shows an important difference between global traces and marginal traces: in the latter case, the right trace may be complete without implying the completeness of the left trace. This explains our use of three conditions $(A C 1, A C 2$ and $A C 3)$ when studying marginal traces instead of the two classical conditions (Ferrers and semitransitivity) used when studying global traces.

The combination of our three conditions $(A C 1, A C 2$ and $A C 3)$ implies that the marginal traces induced by $\succsim$ are weak orders. Unsurprisingly, this implies that marginal relations $\succsim_{i}$ do have special properties even when they differ from marginal traces (which is the general case). We summarize them in the following:

\section{Proposition 3 (Properties of marginal preferences)}

1. If $\succsim$ is reflexive and either $A C 1_{i}$ or $A C 2_{i}$ holds then $\succsim$ is marginally complete and weakly separable for $i \in N$.

2. If $\succsim$ is reflexive and either $A C 1_{i}$ or $A C 2_{i}$ holds then $\succsim_{i}$ is an interval order.

3. If, in addition, $\succsim$ satisfies $A C 3_{i}$ then $\succsim_{i}$ is a semi-order.

\section{ProOF}

Part 1. We give the proof using $A C 1_{i}$, the proof using $A C 2_{i}$ being similar. Using the reflexivity of $\succsim$, we know that $\left(x_{i}, a_{-i}\right) \succsim\left(x_{i}, a_{-i}\right)$ and $\left(y_{i}, a_{-i}\right) \succsim$ $\left(y_{i}, a_{-i}\right)$. Since $A C 1_{i}$ holds, $\succsim_{i}^{+}$is complete so that $x_{i} \succsim_{i}^{+} y_{i}$ or $y_{i} \succsim_{i}^{+} x_{i}$. If

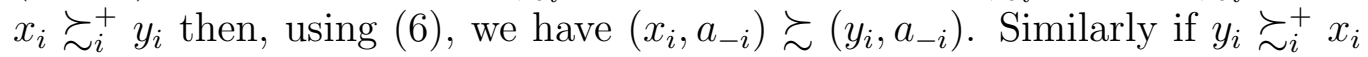
then $\left(y_{i}, a_{-i}\right) \succsim\left(x_{i}, a_{-i}\right)$. Hence, $\succsim$ is marginally complete for $i \in N$.

Suppose now that $\succsim$ is not weakly separable for $i \in N$. Then we have $\left(x_{i}, a_{-i}\right) \succ\left(y_{i}, a_{-i}\right)$ and $\left(y_{i}, b_{-i}\right) \succ\left(x_{i}, b_{-i}\right)$, for some $x_{i}, y_{i} \in X_{i}$ and some $a_{-i}, b_{-i} \in X_{-i}$. Since $\succsim$ is reflexive, we have $\left(y_{i}, a_{-i}\right) \succsim\left(y_{i}, a_{-i}\right)$ and $\left(x_{i}, b_{-i}\right) \succsim\left(x_{i}, b_{-i}\right)$. This would imply $\operatorname{Not}\left[x_{i} \succsim_{i}^{+} y_{i}\right]$ and $\operatorname{Not}\left[y_{i} \succsim_{i}^{+} x_{i}\right]$, violating $A C 1_{i}$. Hence, $\succsim$ is weakly separable for $i \in N$.

Part 2. We know from part 1 that $\succsim$ is marginally complete and weakly separable for $i \in N$. Hence, $\succsim_{i}$ is complete. It remains to prove that $\succsim_{i}$ is Ferrers. It is well-known (Pirlot \& Vincke, 1997, theorem 3.11) that, since $\succsim_{i}$ is complete, the Ferrers condition is equivalent to: $\left[x_{i} \succ_{i} y_{i}, y_{i} \sim_{i} z_{i}\right.$ and $\left.z_{i} \succ_{i} w_{i}\right] \Rightarrow x_{i} \succ_{i} w_{i}$, for all $x_{i}, y_{i}, z_{i}, w_{i} \in X_{i}$. We prove that this condition holds. 
We claim that if $A C 1_{i}$ holds then $\left[x_{i} \sim_{i} z_{i}\right.$ and $\left.x_{i} \succ_{i} y_{i}\right] \Rightarrow z_{i} \succ_{i}^{+} y_{i}$. In contradiction with the thesis, since $\succsim_{i}^{+}$is complete, suppose that $y_{i} \succsim_{i}^{+} z_{i}$. Since $x_{i} \sim_{i} z_{i}$ we have $z_{i} \succsim_{i} x_{i}$ so that $\left(z_{i}, a_{-i}\right) \succsim\left(x_{i}, a_{-i}\right)$, for all $a_{-i} \in X_{-i}$. But $y_{i} \succsim_{i}^{+} z_{i}$ implies $\left(y_{i}, a_{-i}\right) \succsim\left(x_{i}, a_{-i}\right)$, for all $a_{-i} \in X_{-i}$, contradicting $x_{i} \succ_{i} y_{i}$. This proves the claim.

Suppose that $x_{i} \succ_{i} y_{i}, y_{i} \sim_{i} z_{i}$ and $z_{i} \succ_{i} w_{i}$. Using the above claim, we have $y_{i} \succ_{i}^{+} w_{i}$. If $w_{i} \succsim_{i} x_{i}$, so that $\left(w_{i}, a_{-i}\right) \succsim\left(x_{i}, a_{-i}\right)$, for all $a_{-i} \in X_{-i}$, $y_{i} \succ_{i}^{+} w_{i}$ implies $\left(y_{i}, a_{-i}\right) \succsim\left(x_{i}, a_{-i}\right)$, for all $a_{-i} \in X_{-i}$, contradicting $x_{i} \succ_{i} y_{i}$. Hence $x_{i} \succ_{i} w_{i}$. The proof using $A C 2_{i}$ is similar.

Part 3. In view of part 2 above, all we have to show is that $\succsim_{i}$ is semitransitive. It is well-known Pirlot and Vincke (1997, theorem 3.1) that, for a complete relation, semi-transitivity is equivalent to saying that no pair of elements of $X_{i}$ can be linked both by a path of two consecutive $\succ_{i}$-arcs and by a path of two consecutive $\sim_{i}$-arcs, i.e., for all $x_{i}, y_{i}, z_{i}, w_{i} \in X_{i}$, we may not have $x_{i} \succ_{i} y_{i}, y_{i} \succ_{i} z_{i}, x_{i} \sim_{i} w_{i}$ and $w_{i} \sim_{i} z_{i}$. We prove that this condition holds.

Suppose that $x_{i} \succ_{i} y_{i}, y_{i} \succ_{i} z_{i}, x_{i} \sim_{i} w_{i}$ and $w_{i} \sim_{i} z_{i}$. Using $A C 1_{i}$ and the claim proved in part 2 above, we know that this implies $w_{i} \succ_{i}^{+} y_{i}$. In view of part 3 of lemma $3, A C 3_{i}$ then implies $w_{i} \succsim_{i}^{-} y_{i}$. Since $w_{i} \sim_{i} z_{i}$, we have $\left(z_{i}, a_{-i}\right) \succsim\left(w_{i}, a_{-i}\right)$, for all $a_{-i} \in X_{-i}$ and $w_{i} \succsim_{i}^{-} y_{i}$ leads to $\left(z_{i}, a_{-i}\right) \succsim$ $\left(y_{i}, a_{-i}\right)$, for all $a_{-i} \in X_{-i}$, contradicting $y_{i} \succ_{i} z_{i}$. The proof using $A C 2_{i}$ is similar and is omitted.

\subsection{Strict responsiveness to marginal traces}

Keeping in mind the classical constant threshold numerical representation for finite semi-orders (see Pirlot \& Vincke, 1997; Scott \& Suppes, 1958), it is clear that, in general, in a semi-order we may have $x \succsim y, y \succ^{ \pm} z$ and $x \sim z$. Hence, $\succsim$ may not be strictly responsive to $\succ^{ \pm}$even when $\succsim$ and $\succsim^{ \pm}$ are complete. Indeed, it is easy to see that a semi-order for which

$$
\left[x \succsim y \text { and } y \succ^{ \pm} z\right] \Rightarrow x \succ z,
$$

must be a weak order.

Considering marginal traces, it is now possible to envisage binary relations that are strictly responsive to each of their marginal traces without implying that they are (semi-)transitive or Ferrers.

\section{Definition 4 (Condition $A C 4, T A C 1, T A C 2$ )}

We say that $\succsim$ satisfies:

$A C 4_{i}$ if it satisfies $A C 3_{i}$ and when one of the two conclusions of $A C 3_{i}$ is false then the other one holds with $\succ$ instead of $\succsim$, 
$T A C 1_{i}$ if

$$
\left.\begin{array}{c}
\left(x_{i}, a_{-i}\right) \succsim y \\
\text { and } \\
y \succsim\left(z_{i}, a_{-i}\right) \\
\text { and } \\
\left(z_{i}, b_{-i}\right) \succsim w
\end{array}\right\} \Rightarrow\left(x_{i}, b_{-i}\right) \succsim w
$$

$T A C 2_{i}$ if

$$
\left.\begin{array}{l}
\left(x_{i}, a_{-i}\right) \underset{ }{\succsim y} \\
\text { and } \\
y \succsim\left(z_{i}, a_{-i}\right) \\
\text { and } \\
w \succsim\left(x_{i}, b_{-i}\right)
\end{array}\right\} \Rightarrow w \succsim\left(z_{i}, b_{-i}\right),
$$

for all $x_{i}, z_{i} \in X_{i}$, all $a_{-i}, b_{-i} \in X_{-i}$ and all $y, w \in X$.

We say that $\succsim$ satisfies $A C 4$ (resp. TAC1, TAC2) if it satisfies $A C 4_{i}$ (resp. $T A C 1_{i}, T A C 2_{i}$ ) for all $i \in N$.

Condition $A C 4_{i}$ is a clear strengthening of $A C 3_{i}$. As soon as $\succsim$ is reflexive, $A C 4_{i}$ will imply both $A C 1_{i}$ and $A C 2_{i}$. Conditions $T A C 1_{i}$ and $T A C 2_{i}$ (the rationale for the names being that $T A C 1$ and $T A C 2$ are intrA-attribute Cancellation conditions involving Three premises) will prove equivalent to $A C 4_{i}$ when $\succsim$ is complete. The first two premises of $T A C 1_{i}$ and $T A C 2_{i}$ suggest that the level $x_{i}$ is not worse than the level $z_{i}$. TAC $1_{i}$ (resp. TAC2 $2_{i}$ ) then imply than $x_{i}$ should upward dominate (resp. downward dominate) $z_{i}$.

\section{Lemma 4 (Strict responsiveness to marginal traces)}

1. $A C 4_{i}$ is equivalent to $A C 3_{i}$ and the conjunction of the following two conditions:

$$
\begin{aligned}
& x \succsim y \text { and } \operatorname{Not}\left[x_{i} \succsim_{i}^{+} z_{i}\right] \Rightarrow \operatorname{Not}\left[y \succsim_{\left.\left(z_{i}, x_{-i}\right)\right]}\right. \\
& x \succsim y \text { and } \operatorname{Not}\left[w_{i} \succsim_{i}^{-} y_{i}\right] \Rightarrow \operatorname{Not}\left[\left(w_{i}, y_{-i}\right) \succsim x\right] .
\end{aligned}
$$

2. If $\succsim$ is reflexive, $A C 4_{i}$ is equivalent to the completeness of $\succsim_{i}^{ \pm}$and the conjunction of the following two conditions:

$$
\begin{aligned}
& {\left[x \succsim y \text { and } z_{i} \succ_{i}^{ \pm} x_{i}\right] \Rightarrow\left(z_{i}, x_{-i}\right) \succ y,} \\
& {\left[x \succsim y \text { and } y_{i} \succ_{i}^{ \pm} w_{i}\right] \Rightarrow x \succ\left(w_{i}, y_{-i}\right) .}
\end{aligned}
$$

3. If $\succsim$ is reflexive and satisfies $A C 4_{i}$ then

- $\succsim$ is independent for $\{i\}$, 
- ${ }_{i}$ is a weak-order and

- $\succsim_{i}=\succsim_{i}^{ \pm}$.

4. If $\succsim$ is complete, TAC $1_{i}$ is equivalent to the completeness of $\succsim_{i}^{+}$and the following condition:

$$
\left[x \succsim y \text { and } z_{i} \succ_{i}^{+} x_{i}\right] \Rightarrow\left(z_{i}, x_{-i}\right) \succ y
$$

5. If $\succsim$ is complete, TAC2 $2_{i}$ is equivalent to the completeness of $\succsim_{i}^{-}$and the following condition:

$$
\left[x \succsim y \text { and } y_{i} \succ_{i}^{-} w_{i}\right] \Rightarrow x \succ\left(w_{i}, y_{-i}\right) .
$$

6. If $\succsim$ is complete, $\left[T A C 1_{i}\right.$ and $\left.T A C 2_{i}\right] \Leftrightarrow A C 4_{i}$.

7. In the class of complete relations, TAC1 and TAC2 are independent conditions.

8. There are weakly independent semi-orders verifying TAC1 and TAC2 that are not weak orders.

\section{PROOF}

Part 1. $[\Rightarrow]$. By definition, $A C 4_{i}$ implies $A C 3_{i}$. We prove that $\left[A C 4_{i} \Rightarrow\right.$ (11)], the proof for (12) being similar. Suppose that (11) is violated so that $x \succsim y,\left(z_{i}, a_{-i}\right) \succsim w, \operatorname{Not}\left[\left(x_{i}, a_{-i}\right) \succsim w\right]$ and $y \succsim\left(z_{i}, x_{-i}\right)$. Applying $A C 3_{i}$ to $\left(z_{i}, a_{-i}\right) \succsim w$ and $y \succsim\left(z_{i}, x_{-i}\right)$ yields $\left(x_{i}, a_{-i}\right) \succsim w$ or $y \succsim\left(x_{i}, x_{-i}\right)$. Since, by hypothesis, $\operatorname{Not}\left[\left(x_{i}, a_{-i}\right) \succsim w\right], A C 4_{i}$ implies $y \succ x$, a contradiction.

$[\Leftarrow]$. Suppose that $\left(x_{i}, a_{-i}\right) \succsim y$ and $z \succsim\left(x_{i}, b_{-i}\right)$. Using $A C 3_{i}$, we have either $\left(w_{i}, a_{-i}\right) \succsim y$ or $z \succsim\left(w_{i}, d_{-i}\right)$. Suppose, in addition, that $\operatorname{Not}\left[\left(w_{i}, a_{-i}\right) \succsim\right.$ $y]$ and $z \sim\left(w_{i}, d_{-i}\right)$. From $\left(x_{i}, a_{-i}\right) \succsim y$ and $\operatorname{Not}\left[\left(w_{i}, a_{-i}\right) \succsim y\right]$, we know that $\operatorname{Not}\left[w_{i} \succsim_{i}^{+} x_{i}\right]$. Using (11), $\left(w_{i}, d_{-i}\right) \succsim z$ and $N o t\left[w_{i} \succsim_{i}^{+} x_{i}\right]$ imply $\operatorname{Not}\left[z \succsim\left(x_{i}, d_{-i}\right)\right]$, a contradiction. The proof is similar, using (12), if we suppose that: $\left(w_{i}, a_{-i}\right) \sim y$ and $\operatorname{Not}\left[z \succsim\left(w_{i}, d_{-i}\right)\right]$.

Part 2. $[\Rightarrow]$. Let us first show that $\left[A C 4_{i} \Rightarrow A C 1_{i}\right.$ and $\left.A C 2_{i}\right]$ when $\succsim$ is reflexive. Suppose $A C 1_{i}$ is violated so that, for some $x_{i}, z_{i} \in X_{i}, \operatorname{Not}\left[x_{i} \succsim_{i}^{+}\right.$ $\left.z_{i}\right]$ and $\operatorname{Not}\left[z_{i} \succsim_{i}^{+} x_{i}\right]$. Since $A C 3_{i}$ holds, this implies $x_{i} \sim_{i}^{-} z_{i}$. Now, $x \succsim x$ and $\operatorname{Not}\left[x_{i} \succsim_{i}^{+} z_{i}\right]$ imply, using (11), Not $\left[x \succsim\left(z_{i}, x_{-i}\right)\right]$. But $x \succsim x$ and $\operatorname{Not}\left[x \succsim\left(z_{i}, x_{-i}\right)\right]$ imply $\operatorname{Not}\left[x_{i} \succsim_{i}^{-} z_{i}\right]$, a contradiction. The proof for $A C 2_{i}$ using (12) is similar. Hence, $A C 1_{i}$ and $A C 2_{i}$ hold. Since $A C 3_{i}$ holds by construction, $\succsim_{i}^{ \pm}$is complete.

Let us now show that (13) holds. Suppose that $x \succsim y$ and $z_{i} \succ_{i}^{ \pm} x_{i}$. From the definition of $\succsim_{i}^{ \pm}$we know that $\left(z_{i}, x_{-i}\right) \succsim y$. Suppose now that, in 
contradiction with the thesis, $y \succsim\left(z_{i}, x_{-i}\right)$. Since $\succsim_{i}^{ \pm}$is complete, $z_{i} \succ_{i}^{ \pm} x_{i}$ implies either $\operatorname{Not}\left[x_{i} \succsim_{i}^{+} z_{i}\right]$ or $\operatorname{Not}\left[x_{i} \succsim_{i}^{-} z_{i}\right]$. If $\operatorname{Not}\left[x_{i} \succsim_{i}^{+} z_{i}\right]$, then, using (11), $x \succsim y$ would imply $\operatorname{Not}\left[y \succsim\left(z_{i}, x_{-i}\right)\right]$, a contradiction. Similarly if $\operatorname{Not}\left[x_{i} \succsim_{i}^{-} z_{i}\right], y \succsim\left(z_{i}, x_{-i}\right)$ would imply, using $(12), \operatorname{Not}[x \succsim y]$, a contradiction. The proof for (14) is similar.

$[\Leftarrow]$. Since $\succsim_{i}^{ \pm}$is complete, we know that $A C 3_{i}$ holds. We show that the part of $A C 4_{i}$ not covered by $A C 3_{i}$ holds. Suppose that $\left(x_{i}, a_{-i}\right) \succsim y$, $z \succsim\left(x_{i}, b_{-i}\right), \operatorname{Not}\left[\left(w_{i}, a_{-i}\right) \succsim y\right]$ and $z \sim\left(w_{i}, b_{-i}\right)$. From $\left(x_{i}, a_{-i}\right) \succsim y$ and $\operatorname{Not}\left[\left(w_{i}, a_{-i}\right) \succsim y\right]$, we know that $\operatorname{Not}\left[w_{i} \succsim_{i}^{+} x_{i}\right]$, so that $x_{i} \succ_{i}^{ \pm} w_{i}$. Using (13), $\left(w_{i}, b_{-i}\right) \succsim z$ would imply $\left(x_{i}, b_{-i}\right) \succ z$, a contradiction. The proof is similar, using (14), if $\left(w_{i}, a_{-i}\right) \sim y$ and $\operatorname{Not}\left[z \succsim\left(w_{i}, b_{-i}\right)\right]$.

Part 3. Suppose that $\left(x_{i}, a_{-i}\right) \succsim\left(y_{i}, a_{-i}\right)$ and $\operatorname{Not}\left[\left(x_{i}, b_{-i}\right) \succsim\left(y_{i}, b_{-i}\right)\right]$. Since $\succsim$ is reflexive, we know that $\left(y_{i}, b_{-i}\right) \succsim\left(y_{i}, b_{-i}\right)$. Thus, since we know from part 2 that $\succsim_{i}^{ \pm}$is complete, we have $y_{i} \succ_{i}^{ \pm} x_{i}$. Using (13), $y_{i} \succ_{i}^{ \pm} x_{i}$ and $\left(x_{i}, a_{-i}\right) \succsim\left(y_{i}, a_{-i}\right)$ would imply $\left(y_{i}, a_{-i}\right) \succ\left(y_{i}, a_{-i}\right)$, a contradiction. Hence, $\succsim$ is independent for $\{i\}$.

Since $\succsim$ is reflexive, we know, from part 2 that $\succsim_{i}^{ \pm}$is complete. Using reflexivity and (8), we have: $x_{i} \succsim_{i}^{ \pm} y_{i} \Rightarrow x_{i} \succsim_{i} y_{i}$. Let us show that $x_{i} \succ_{i}^{ \pm}$ $y_{i} \Rightarrow x_{i} \succ_{i} y_{i}$, which will complete the proof. Suppose that $x_{i} \succ_{i}^{ \pm} y_{i}$. Since $\succsim$ is reflexive, we have $\left(y_{i}, a_{-i}\right) \succsim\left(y_{i}, a_{-i}\right)$, for all $a_{-i} \in X_{-i}$. Using (13), we obtain $\left(x_{i}, a_{-i}\right) \succ\left(y_{i}, a_{-i}\right)$, for all $a_{-i} \in X_{-i}$. We thus have $x_{i} \succ_{i} y_{i}$.

Part 4. $[\Rightarrow]$. Let us first show that when $\succsim$ is complete, $T A C 1_{i} \Rightarrow$ $A C 1_{i}$. Suppose that $A C 1_{i}$ is violated so that $\left(x_{i}, a_{-i}\right) \succsim y,\left(z_{i}, b_{-i}\right) \succsim w$ $\operatorname{Not}\left[\left(z_{i}, a_{-i}\right) \succsim y\right]$ and $\operatorname{Not}\left[\left(x_{i}, b_{-i}\right) \succsim w\right]$. Since $\succsim$ is complete, we know that $y \succsim\left(z_{i}, a_{-i}\right)$. Using TAC1 $1_{i},\left(x_{i}, a_{-i}\right) \succsim y, y \succsim\left(z_{i}, a_{-i}\right)$ and $\left(z_{i}, b_{-i}\right) \succsim w$ imply $\left(x_{i}, b_{-i}\right) \succsim w$, a contradiction. Hence $A C 1_{i}$ holds and $\succsim_{i}^{+}$is complete.

Suppose now, in contradiction with (15) that $x \succsim y, z_{i} \succ_{i}^{+} x_{i}$ and $y \succsim$ $\left(z_{i}, x_{-i}\right)$. We know that $\operatorname{Not}\left[x_{i} \succsim_{i}^{+} z_{i}\right]$, so that $\left(z_{i}, a_{-i}\right) \succsim w$ and $w \succ\left(x_{i}, a_{-i}\right)$, for some $w \in X$ and some $a_{-i} \in X_{-i}$. Using $T A C 1_{i}, x \succsim y, y \succsim\left(z_{i}, x_{-i}\right)$ and $\left(z_{i}, a_{-i}\right) \succsim w$ imply $\left(x_{i}, a_{-i}\right) \succsim w$, a contradiction.

$[\Leftarrow]$. Suppose that $T A C 1_{i}$ is violated so that $\left(x_{i}, a_{-i}\right) \succsim y, y \succsim\left(z_{i}, a_{-i}\right)$ $\left(z_{i}, b_{-i}\right) \succsim w$ and $w \succ\left(x_{i}, b_{-i}\right)$. This implies $\operatorname{Not}\left[x_{i} \succsim_{i}^{+} z_{i}\right]$. Since $\succsim_{i}^{+}$is complete, we have $z_{i} \succ_{i}^{ \pm} x_{i}$. Using (15), $\left(x_{i}, a_{-i}\right) \succsim y$ and $z_{i} \succ_{i}^{ \pm} x_{i}$ would imply $\left(z_{i}, a_{-i}\right) \succ y$, a contradiction.

The proof of part 5 is similar.

Part 6 . $[\Rightarrow]$. In view of parts 2,4 and 5 , all we have to show is that $\succsim_{i}^{ \pm}$ is complete, i.e. that $A C 3_{i}$ holds.

Suppose that $A C 3_{i}$ is violated so that $\left(x_{i}, a_{-i}\right) \succsim y, w \succsim\left(x_{i}, b_{-i}\right)$, $\operatorname{Not}\left[\left(z_{i}, a_{-i}\right) \succsim y\right]$ and $\operatorname{Not}\left[w \succsim\left(z_{i}, b_{-i}\right)\right]$, for some $x_{i}, z_{i} \in X_{i}, a_{-i}, b_{-i} \in X_{-i}$ and $y, w \in X$. Since $\succsim$ is complete, we have $\left(z_{i}, b_{-i}\right) \succsim w$. Using $T A C 1_{i}$, $\left(z_{i}, b_{-i}\right) \succsim w, w \succsim\left(x_{i}, b_{-i}\right)$ and $\left(x_{i}, a_{-i}\right) \succsim y$ imply $\left(z_{i}, a_{-i}\right) \succsim y$, a contradic- 
tion.

$[\Leftarrow]$. We show that $A C 4_{i} \Rightarrow T A C 1_{i}$, the proof for $T A C 2_{i}$ being similar. Suppose that $T A C 1_{i}$ is violated so that $\left(x_{i}, a_{-i}\right) \succsim y, y \succsim\left(z_{i}, a_{-i}\right),\left(z_{i}, b_{-i}\right) \succsim$ $w$ and $w \succ\left(x_{i}, b_{-i}\right)$. This implies, since $\succsim_{i}^{ \pm}$is complete, $z_{i} \succ_{i}^{ \pm} x_{i}$. Using (13), $\left(x_{i}, a_{-i}\right) \succsim y$ and $z_{i} \succ_{i}^{ \pm} x_{i}$ would imply $\left(z_{i}, a_{-i}\right) \succ y$, a contradiction.

Parts 7 and 8: see examples 4 and 5 in appendix A.

As soon as $\succsim$ is reflexive, condition $A C 4_{i}$ is therefore exactly what is needed to ensure the strict responsiveness of $\succsim$ with respect to $\succ_{i}^{ \pm}$. This also implies that $\succsim$ is independent for $\{i\}$ and that $\succsim_{i}=\succsim_{i}^{ \pm}$. Note that, while $A C 4_{i}$ implies that $\succsim$ is strictly responsive to $\succsim_{i}^{ \pm}$, it does not imply that it is (semi-)transitive or Ferrers. When $\succsim$ is complete, condition $A C 4_{i}$ can be factorized as the conjunction of $T A C 1_{i}$ and $T A C 2_{i}$. Using (13) and (14) (resp. (15) and (16)) can facilitate the test of $A C 4_{i}$ (resp. TAC1 $1_{i}$ and $\left.T A C 2_{i}\right)$.

\section{Relations compatible with dominance}

A binary relation $\succsim$ on a set $X=\prod_{i=1}^{n} X_{i}$ is said to be compatible with a dominance relation if it possible to define a weak order on each $X_{i}$ in such a way that these weak orders "combine nicely" with $\succsim$. The intuitive idea underlying the following definition is that, if $z$ (resp. $y$ ) is judged "at least as good" as $x$ (resp. $w$ ) using the weak orders on each attribute and if $x \succsim y$ then it should follow that $z \succsim w$. Note that we only define below dominance-compatibility for reflexive binary relations, interpreting $\succsim$ as an "at least good as" preference relation between alternatives. Although it is not difficult to study the case of asymmetric binary relations, we do not investigate this point here.

\section{Definition 5 (Dominance-compatible relations)}

$A$ reflexive binary relation $\succsim$ on a set $X=\prod_{i=1}^{n} X_{i}$ is compatible with a dominance relation if, for all $i \in N$, there is a weak order $S_{i}$ on $X_{i}$ such that, for all $x, y \in X$ and all $z_{i}, w_{i} \in X_{i}$,

$$
\left[x \succsim y, z_{i} S_{i} x_{i} \text { and } y_{i} S_{i} w_{i} \text { for all } i \in N\right] \Rightarrow z \succsim w .
$$

This compatibility is said to be strict when the conclusion of condition (17) is modified to $z \succ w$ as soon as $z_{j} P_{j} x_{j}$ or $y_{j} P_{j} w_{j}$ for some $j \in N$, where $P_{j}$ denotes the asymmetric part of $S_{j}$.

Intuition might suggest that an alternative definition of dominance-compatibility would consist in requiring that:

$$
\left[x_{i} S_{i} y_{i} \text { for all } i \in N\right] \Rightarrow x \succsim y .
$$


It is easy to convince oneself that such a definition is too weak to capture the whole idea of dominance when $\succsim$ is not supposed to be complete or transitive. Indeed, it is easy to see that when $\succsim$ has cycles in its asymmetric part, it might obey (18) while there may exist $x, y, z \in X$ such that $x \Delta y, y \succ z$ and $z \succ x$ (where $\Delta$ denotes the dominance relation, i.e. $x \Delta y \Leftrightarrow x_{i} S_{i} y_{i}$ for all $i \in N)$. In such a case, the search for efficient alternatives would be of little help so that it seems difficult to say that $\succsim$ is compatible with dominance.

The definition of dominance-compatibility used here is similar to the one used in Roy (1996), Roy and Bouyssou (1993), Vincke (1992), when defining the notion of a "consistent family of criteria". It clearly implies (18) since $\succsim$ is reflexive. It should be noted that condition (17), which requires that $S_{i}$ combines nicely with $\succsim$, also implies that $S_{i}$ combines nicely with $\succ$. It is easy to see that condition (17) implies that:

$$
\left[x \succ y, z_{i} S_{i} x_{i} \text { and } y_{i} S_{i} w_{i} \text {, for all } i \in N\right] \Rightarrow z \succ w .
$$

From the preceding section, it is expected that if a binary relation $\succsim$ is dominance-compatible, the weak orders $S_{i}$ on each attribute should be closely linked to the marginal traces induced by $\succsim$ on each $X_{i}$. Similarly it is also expected that strict compatibility with dominance should be related with the strict responsiveness of $\succsim$ to its marginal traces. As shown below this is indeed the case.

\section{Theorem 1 (Dominance-compatibility)}

$A$ reflexive binary relation $\succsim$ on a set $X=\prod_{i=1}^{n} X_{i}$ is

1. compatible with a dominance relation if and only if it satisfies $A C 1$, $A C 2$ and $A C 3$,

2. strictly compatible with a dominance relation if and only if it satisfies $A C 4$.

\section{PROOF}

Part 1. The necessity of $A C 1, A C 2$ and $A C 3$ is easily shown. We take the example of $A C 1$, the other cases being similar. Suppose that $\left(x_{i}, a_{-i}\right) \succsim y$ and $\left(z_{i}, b_{-i}\right) \succsim w$. The relation $S_{i}$ being complete, we have either $x_{i} S_{i} z_{i}$ or $z_{i} S_{i} x_{i}$. If $z_{i} S_{i} x_{i}$ then, using the definition of dominance compatibility, $\left(x_{i}, a_{-i}\right) \succsim y$ implies $\left(z_{i}, a_{-i}\right) \succsim y$. If $x_{i} S_{i} z_{i}$, then $\left(z_{i}, b_{-i}\right) \succsim w$ implies $\left(x_{i}, b_{-i}\right) \succsim w$. Hence $A C 1$ holds.

The sufficiency of $A C 1, A C 2$ and $A C 3$ is obvious, in view of part 4 of lemma 3 and (8), letting $S_{i}=\succsim_{i}^{ \pm}$for all $i \in N$.

Part 2 . When $\succsim$ is reflexive, we know from part 2 of lemma 4 that $A C 4_{i}$ implies all of $A C 1_{i}, A C 2_{i}$ and $A C 3_{i}$. In view of part 1 above, we only have 
to show the necessity of the part of $A C 4_{i}$ not covered by $A C 3_{i}$. Suppose that $z \succsim\left(x_{i}, a_{-i}\right)$ and $\left(x_{i}, b_{-i}\right) \succsim y$. The relation $S_{i}$ being complete, we have either $x_{i} I_{i} w_{i}, x_{i} P_{i} w_{i}$ or $w_{i} P_{i} x_{i}$, where $I_{i}$ and $P_{i}$ respectively denote the symmetric and asymmetric part of $S_{i}$. If $x_{i} I_{i} w_{i}$ then, using the definition of dominancecompatibility, $z \succsim\left(w_{i}, a_{-i}\right)$ and $\left(w_{i}, b_{-i}\right) \succsim y$, so that there is nothing to prove. If $x_{i} P_{i} w_{i}$ then, using the definition of strict dominance-compatibility, we obtain $z \succ\left(w_{i}, a_{-i}\right)$. Similarly, if $w_{i} P_{i} x_{i}$, we obtain $\left(w_{i}, b_{-i}\right) \succ y$. Thus $A C 4_{i}$ holds.

The sufficiency of $A C 4$ results from part 1 above and part 2 of lemma 4 , letting $S_{i}=\succsim_{i}^{ \pm}$for all $i \in N$.

Within a conjoint measurement framework, theorem 1 gives necessary and sufficient conditions for a binary relation to be (strictly) dominancecompatible. It should be noticed that these conditions do not imply that $\succsim$ is complete or has "nice" transitivity properties. In fact using examples inspired from Condorcet's paradox (see e.g. Sen (1986)), it is easy to build a strictly dominance-compatible binary relation $\succsim$ having circuits in its asymmetric part (e.g. building $\succsim$ via the simple majority method applied to the relations $\left.S_{i}\right)$.

Let us note that if a binary relation $\succsim$ is strictly compatible with a dominance relation, the weak orders $S_{i}$ are necessarily unique (indeed suppose that there are two distinct such families of weak orders $S_{i}$ and $S_{i}^{\prime}$; then $x_{i} P_{i} y_{i}$ and $y_{i} S_{i}^{\prime} x_{i}$ would imply, using the reflexivity of $\succsim$, both $\left(x_{i}, x_{-i}\right) \succ\left(y_{i}, x_{-i}\right)$ and $\left.\left(y_{i}, x_{-i}\right) \succsim\left(x_{i}, x_{-i}\right)\right)$. This is not so when only dominance-compatibility is required since elements in the same equivalence class of $\sim_{i}^{ \pm}$may be ranked in whatever order by $S_{i}$. It is nevertheless easy to see that we always have:

$$
x_{i} \succ_{i}^{ \pm} y_{i} \Rightarrow x_{i} P_{i} y_{i}
$$

so that $S_{i}$ are unique on $X_{i} / \sim_{i}^{ \pm}$.

When $\succsim$ is complete, it is clearly possible to combine part 6 of lemma 4 with theorem 1 to modify the characterization of strict compatibility with dominance using TAC1 and TAC2 instead of $A C 4$.

It is worth noting at that point that the characterization of (strict) compatibility with a dominance relation can be greatly simplified when $\succsim$ is a weak order. The case of weak orders is indeed highly specific since, in that case, we know that the global trace $\succsim^{ \pm}$is equal to $\succsim$ and the marginal trace $\succsim_{i}^{ \pm}$is equal to the marginal preference $\succsim_{i}$.

\section{Lemma 5 (Dominance and weak orders)}

Let $\succsim$ be weak order on a set $X=\prod_{i=1}^{n} X_{i}$. Then: 
1. $[\succsim$ is weakly separable $] \Leftrightarrow[\succsim$ satisfies $A C 1] \Leftrightarrow[\succsim$ satisfies $A C 2] \Leftrightarrow[\succsim$ satisfies $A C 3]$,

2. $[\succsim$ is weakly independent $] \Leftrightarrow[\succsim$ satisfies $A C 4]$.

\section{ProOF}

Part 1 . We show that, when $\succsim$ is a weak order, weak separability holds if and only if $A C 1$ holds. The proof of the other equivalences is similar.

$[A C 1 \Rightarrow$ Weak separability]. Suppose that $\succsim$ is not weakly separable. Therefore there is an $i \in N$ and $x_{i}, y_{i} \in X_{i}$ such that $\left(x_{i}, z_{-i}\right) \succ\left(y_{i}, z_{-i}\right)$ and $\left(y_{i}, w_{-i}\right) \succ\left(x_{i}, w_{-i}\right)$, for some $z_{-i}, w_{-i} \in X_{-i}$. Since $\succsim$ is reflexive, we have $\left(x_{i}, z_{-i}\right) \succsim\left(x_{i}, z_{-i}\right)$ and $\left(y_{i}, w_{-i}\right) \succsim\left(y_{i}, w_{-i}\right)$. Using $A C 1$, we have either

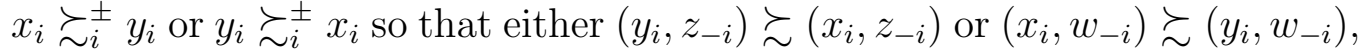
a contradiction.

[Weak separability $\Rightarrow A C 1$ ]. Suppose that $A C 1$ is violated so that, since $\succsim$ is complete, $\left(x_{i}, a_{-i}\right) \succsim y,\left(z_{i}, c_{-i}\right) \succsim w, y \succ\left(z_{i}, a_{-i}\right)$ and $w \succ\left(x_{i}, c_{-i}\right)$, for some $x_{i}, z_{i} \in X_{i}$, some $a_{-i}, c_{-i} \in X_{-i}$ and some $y, w \in X$. Since $\succsim$ is a weak order, we obtain $\left(x_{i}, a_{-i}\right) \succ\left(z_{i}, a_{-i}\right)$ and $\left(z_{i}, c_{-i}\right) \succ\left(x_{i}, c_{-i}\right)$, which violates weak separability.

Part 2. $\quad[A C 4 \Rightarrow$ Weak independence]. Suppose that $\succsim$ is not weakly independent, i.e. there is an $i \in N$ and $x_{i}, y_{i} \in X_{i}$ such that $\left(x_{i}, z_{-i}\right) \succsim$ $\left(y_{i}, z_{-i}\right)$ and $\left(y_{i}, w_{-i}\right) \succ\left(x_{i}, w_{-i}\right)$ for some $z_{-i}, w_{-i} \in X_{-i}$. Since $\succsim$ is reflexive we have $\left(x_{i}, z_{-i}\right) \succsim\left(x_{i}, z_{-i}\right)$ and $\left(x_{i}, w_{-i}\right) \succsim\left(x_{i}, w_{-i}\right)$. Using $A C 3$ we must have either $\left(y_{i}, z_{-i}\right) \succsim\left(x_{i}, z_{-i}\right)$ or $\left(x_{i}, w_{-i}\right) \succsim\left(y_{i}, w_{-i}\right)$. The second condition being false by hypothesis, $A C 4$ implies $\left(y_{i}, z_{-i}\right) \succ\left(x_{i}, z_{-i}\right)$, a contradiction.

[Weak independence $\Rightarrow A C 4$ ]. In view of part 1 above, we only have to show the necessity of the part of $A C 4$ not covered by $A C 3$. Suppose, using the completeness of $\succsim$, that $\left(x_{i}, a_{-i}\right) \succsim y, w \succsim\left(x_{i}, b_{-i}\right)$ and either $\left[y \succ\left(z_{i}, a_{-i}\right)\right.$ and $\left.w \sim\left(z_{i}, b_{-i}\right)\right]$ or $\left[\left(z_{i}, a_{-i}\right) \sim y\right.$ and $\left.\left(z_{i}, b_{-i}\right) \succ w\right]$. We deal with the first case, the other one being similar. We have $y \succ\left(z_{i}, a_{-i}\right)$ and $\left(x_{i}, a_{-i}\right) \succsim y$, which imply, since $\succsim$ is a weak order, $\left(x_{i}, a_{-i}\right) \succ\left(z_{i}, a_{-i}\right)$. Similarly, $w \succsim\left(x_{i}, b_{-i}\right)$ and $w \sim\left(z_{i}, b_{-i}\right)$ imply $\left(z_{i}, b_{-i}\right) \succsim\left(x_{i}, b_{-i}\right)$, which violates weak independence.

As show by examples 1 to 3 in appendix A, it is not possible to simplify the characterization of dominance-compatibility in a similar way for semiorders. Indeed, there are weakly independent semi-orders which may violate $A C 1, A C 2$ or $A C 3$. Again, this shows that the case of weak orders is highly specific. 


\section{Traces and numerical representations}

\subsection{Background}

Following the strategy of Bouyssou and Pirlot (2002a) we shall use very general numerical representations as a guideline for our study. We recall here some well-known facts about trivial numerical representations of binary relations on sets without special structure. Although the results in this section may be part of the folklore of binary relations (see Ebert, 1985), we outline their proof, the logic of which being useful in the sequel.

In order to concentrate on the core arguments, we suppose here that $A$ is countable (i.e. finite or countably infinite). The general case is studied in appendix B.

Let $\succsim$ be a binary relation of a set $A$. It is clearly always possible to build a, trivial, numerical representation of $\succsim$ such that:

$$
a \succsim b \Leftrightarrow \mathcal{G}(a, b) \geq 0
$$

where $\mathcal{G}$ is a real-valued function on $A^{2}$ defined letting, for all $a, b \in A$ :

$$
\mathcal{G}(a, b)= \begin{cases}+1 & \text { if } a \succsim b \\ -1 & \text { otherwise }\end{cases}
$$

It is possible to further specify the trivial numerical representation (20). Remember that we defined an equivalence relation $\sim^{ \pm}$on the basis of $\succsim$. Since $A$ is countable (in fact, as soon as the cardinality of $A / \sim^{ \pm}$is not "too large"), there is a real-valued function $u$ on $A$ such that, for all $a, b \in A$ :

$$
a \sim^{ \pm} b \Leftrightarrow u(a)=u(b) .
$$

As shown below, such a function can be integrated in a numerical representation of type (20).

\section{Proposition 4 (Trivial numerical representations)}

Let $\succsim$ be a binary relation on a countable set $A$.

1. There is a real-valued function $u$ on $A$ and a real-valued function $\mathcal{F}$ on $u(A)^{2}$ such that, for all $a, b \in A$ :

$$
a \succsim b \Leftrightarrow \mathcal{F}(u(a), u(b)) \geq 0,
$$

2. The function $\mathcal{F}$ in (22) can be chosen so that $\mathcal{F}(\alpha, \alpha) \geq 0$, for all $\alpha \in u(A)$, if and only if $\succsim$ is reflexive, 
3. The function $\mathcal{F}$ in (22) can be chosen so as to be skew symmetric (i.e. $\mathcal{F}(\alpha, \beta)=-\mathcal{F}(\beta, \alpha)$, for all $\alpha, \beta \in u(A))$ if and only if $\succsim$ is complete.

\section{PROOF}

Part 1. Take any function $u$ satisfying $(21)$ and define $\mathcal{F}$ letting, for all $a, b \in A$ :

$$
\mathcal{F}(u(a), u(b))= \begin{cases}+1 & \text { if } a \succsim b, \\ -1 & \text { otherwise. }\end{cases}
$$

We have to show that $\mathcal{F}$ is well-defined, i.e. that $[u(a)=u(c)$ and $u(b)=u(d)]$ implies $[a \succsim b \Leftrightarrow c \succsim d]$. This is (5). The proof of part 2 is obvious.

Part 3. Take any function $u$ satisfying $(21)$ and define $\mathcal{F}$ letting, for all $a, b \in A$ :

$$
\mathcal{F}(u(a), u(b))=\left\{\begin{aligned}
+1 & \text { if } a \succ b, \\
0 & \text { if } a \sim b, \\
-1 & \text { otherwise. }
\end{aligned}\right.
$$

Using the completeness of $\succsim$ and (5), it is easy to see that $\mathcal{F}$ is well-defined and skew symmetric. The converse is immediate.

Requiring some monotonicity properties linking $\mathcal{F}$ and $u$ in representation (22) unsurprisingly leads to much more constrained structures. We have:

\section{Proposition 5 (Semi-orders and weak orders)}

Let $\succsim$ be a binary relation on countable set $A$. Then:

1. $\succsim$ has a representation of type (22) with $\mathcal{F}$ increasing in its first argument and decreasing in its second argument if and only if $\succsim$ is Ferrers and semi-transitive.

2. $\succsim$ has a representation of type (22) with $\mathcal{F}$ skew symmetric, nondecreasing in its first argument and nonincreasing in its second argument if and only if $\succsim$ is a semi-order,

3. $\succsim$ has a representation of type (22) with $\mathcal{F}$ skew symmetric, increasing in its first argument and decreasing in its second argument if and only if $\succsim$ is a weak order. In that case, it is always possible to take $\mathcal{F}(\alpha, \beta)=$ $\alpha-\beta$.

\section{ProOF}

Part 1. The necessity of Ferrers and semi-transitivity is easily established using the properties of $\mathcal{F}$. Let us for instance show that $\succsim$ is semi-transitive. Suppose that $a \succsim b$ and $b \succsim c$. Hence $\mathcal{F}(u(a), u(b)) \geq 0$ and $\mathcal{F}(u(b), u(c)) \geq$ 0 . If $u(b) \geq u(d)$ then $\mathcal{F}(u(a), u(d)) \geq \mathcal{F}(u(a), u(b)) \geq 0$ so that $a \succsim d$. Otherwise we have $u(d)>u(b)$, which implies $\mathcal{F}(u(d), u(c))>\mathcal{F}(u(b), u(c)) \geq 0$ so that $d \succsim c$. 
In order to show sufficiency, remember from part 2 of proposition 2 that, when $\succsim$ is Ferrers and semi-transitive, $\succsim^{ \pm}$is a weak order. Since $A$ is countable, there is a real-valued function $u$ such that, for all $a, b \in A$ :

$$
a \succsim^{ \pm} b \Leftrightarrow u(a) \geq u(b) .
$$

Using any function $u$ satisfying (25), define $\mathcal{F}$ letting, for all $a, b \in A$,

$$
\mathcal{F}(u(a), u(b))= \begin{cases}+\exp (u(a)-u(b)) & \text { if } a \succsim b, \\ -\exp (u(b)-u(a)) & \text { otherwise. }\end{cases}
$$

That $\mathcal{F}$ is well-defined follows from (5). Its monotonicity properties follow from (4) and its definition.

Part 2. The necessity of completeness, Ferrers and semi-transitivity is easily established.

Sufficiency. Since $\succsim$ is Ferrers and semi-transitive and $A$ is countable, there is a function $u$ satisfying (25). Using any such function $u$, define $\mathcal{F}$ as in (24). That $\mathcal{F}$ is well-defined follows from part 3 of proposition 4 since $\sim^{ \pm}$is the symmetric part of $\succsim^{ \pm}$. The skew symmetry of $\mathcal{F}$ follows from the completeness of $\succsim$. The monotonicity properties of $\mathcal{F}$ follow from (4).

Part 3. The necessity of completeness is obvious. Suppose that $a \succsim b$ and $b \succsim c$. Hence $\mathcal{F}(u(a), u(b)) \geq 0$ and $\mathcal{F}(u(b), u(c)) \geq 0$. Since $\mathcal{F}$ is skew symmetric we know that $\mathcal{F}(u(c), u(b)) \leq 0$. Using the increasingness of $\mathcal{F}, \mathcal{F}(u(a), u(b)) \geq 0$ and $\mathcal{F}(u(c), u(b)) \leq 0$ imply $u(a) \geq u(c)$. Since $\mathcal{F}(u(a), u(a))=0$, because $\mathcal{F}$ is skew symmetric, we have $\mathcal{F}(u(a), u(c)) \geq 0$ so that $a \succsim c$. Hence, $\succsim$ is transitive.

Sufficiency. Since $\succsim$ is a weak order and $A$ is countable, there is a function $u$ such that, for all $a, b \in A$ :

$$
a \succsim b \Leftrightarrow u(a) \geq u(b)
$$

Using any such function $u$, define $\mathcal{F}$ letting, for all $a, b \in A, \mathcal{F}(u(a), u(b))=$ $u(a)-u(b)$.

When $A$ is a product set, it is possible to use the marginal traces of $\succsim$ much in the same way we have just used the global trace $\succsim^{ \pm}$in order to obtain numerical representations. This is explored in what follows.

\subsection{Trivial numerical representations on product sets}

Arbitrary binary relations on product sets have trivial numerical representations of many different kinds (see Bouyssou \& Pirlot, 2002a, 2002b). We 
present one below that will be easily compared with the general representations introduced above. Again, we suppose in this section that $X=\prod_{i=1}^{n} X_{i}$ is countable, the general case being studied in appendix B. We abuse notation in the sequel, writing $F\left(\left[u_{i}\left(x_{i}\right)\right] ;\left[u_{i}\left(y_{i}\right)\right]\right)$ instead of $F\left(u_{1}\left(x_{1}\right), u_{2}\left(x_{2}\right), \ldots\right.$, $\left.u_{n}\left(x_{n}\right), u_{1}\left(y_{1}\right), u_{2}\left(y_{2}\right), \ldots, u_{n}\left(y_{n}\right)\right)$ when there is no risk of confusion.

Proposition 6 (Trivial numerical representations on product sets) Let $\succsim$ be a binary relation on a countable set $X=\prod_{i=1}^{n} X_{i}$. There are realvalued functions $u_{i}$ on $X_{i}$ and a real-valued function $F$ on $\left[\prod_{i=1}^{n} u_{i}\left(X_{i}\right)\right]^{2}$ such that, for all $x, y \in X$ :

$$
x \succsim y \Leftrightarrow F\left(\left[u_{i}\left(x_{i}\right)\right] ;\left[u_{i}\left(y_{i}\right)\right]\right) \geq 0 .
$$

Furthermore, the function $F$ in (27) can be taken so that, for all $x, y \in X$,

1. $F\left(\left[u_{i}\left(x_{i}\right)\right] ;\left[u_{i}\left(x_{i}\right)\right]\right) \geq 0$ iff $\succsim$ is reflexive,

2. $F\left(\left[u_{i}\left(x_{i}\right)\right] ;\left[u_{i}\left(y_{i}\right)\right]\right)=-F\left(\left[u_{i}\left(y_{i}\right)\right] ;\left[u_{i}\left(x_{i}\right)\right]\right)$ iff $\succsim$ is complete.

\section{PROOF}

Let $i \in N$. By construction, $\sim_{i}^{ \pm}$is an equivalence being a reflexive, symmetric and transitive binary relation. Since $X_{i}$ is countable, we know that there is a real-valued function $u_{i}$ on $X_{i}$ such that, for all $x_{i}, y_{i} \in X_{i}$ :

$$
x_{i} \sim_{i}^{ \pm} y_{i} \Leftrightarrow u_{i}\left(x_{i}\right)=u_{i}\left(y_{i}\right)
$$

For each $i \in N$, consider any real-valued function $u_{i}$ on $X_{i}$ satisfying (28). Define $F$ on $\left[\prod_{i=1}^{n} u_{i}\left(X_{i}\right)\right]^{2}$ letting, for all $x, y \in X$,

$$
F\left(\left[u_{i}\left(x_{i}\right)\right] ;\left[u_{i}\left(y_{i}\right)\right]\right)= \begin{cases}+1 & \text { if } x \succsim y \\ -1 & \text { otherwise }\end{cases}
$$

The well-definedness of $F$ follows from (9). The impact of reflexivity on the above representation is obvious.

In order to deal with the "skew symmetric" case $\left(F\left(\left[u_{i}\left(x_{i}\right)\right] ;\left[u_{i}\left(y_{i}\right)\right]\right)=\right.$ $\left.-F\left(\left[u_{i}\left(y_{i}\right)\right] ;\left[u_{i}\left(x_{i}\right)\right]\right)\right)$, consider, for each $i \in N$, a real-valued function $u_{i}$ on $X_{i}$ satisfying (28) and define $F$ on $\left[\prod_{i=1}^{n} u_{i}\left(X_{i}\right)\right]^{2}$ letting, for all $x, y \in X$,

$$
F\left(\left[u_{i}\left(x_{i}\right)\right] ;\left[u_{i}\left(y_{i}\right)\right]\right)=\left\{\begin{aligned}
+1 & \text { if } x \succ y, \\
0 & \text { if } x \sim y, \\
-1 & \text { otherwise }
\end{aligned}\right.
$$

The well-definedness of $F$ follows from (9).

The above proposition is the counterpart of proposition 4 taking the underlying product structure of the set of objects into account. 


\subsection{Marginal traces and numerical representations}

In proposition 6 , the role of $u_{i}$ is merely to attach a number to each equivalence class of $X_{i} / \sim_{i}^{ \pm}$while $F$ passively recodes as +1 's and -1 's (eventually making use of 0 in the skew symmetric case) the presence or absence of $\succsim$ for every possible combination of elements of $X_{i} / \sim_{i}^{ \pm}$. Clearly, as was the case in section 5.1, the situation radically changes as soon as $F$ is supposed to have some monotonicity properties w.r.t. the $u_{i}$ 's. The, important, difference here is that these additional properties do not imply that $\succsim$ is complete, Ferrers or (semi-)transitive.

\section{Theorem 2 (Numerical representations on product sets)}

Let $\succsim$ be a binary relation on a countable set $X=\prod_{i=1}^{n} X_{i}$. There is a numerical representation of type (27) in which $F$ is increasing in its first $n$ arguments and decreasing in its last $n$ arguments iff $\succsim$ satisfies $A C 1, A C 2$ and $A C 3$. In addition, $F$ can be taken so that $F\left(\left[u_{i}\left(x_{i}\right)\right] ;\left[u_{i}\left(x_{i}\right)\right]\right) \geq 0$ iff $\succsim$ is reflexive.

\section{PROOF}

The necessity of $A C 1, A C 2$ and $A C 3$ is easily shown using the properties of $F$. We take the case of $A C 3$. Suppose that $\left(x_{i}, a_{-i}\right) \succsim y$ and $w \succsim\left(x_{i}, b_{-i}\right)$ so that, abusing notation, $F\left(\left[u_{i}\left(x_{i}\right), u_{j}\left(a_{j}\right)_{j \neq i}\right] ;\left[u_{j}\left(y_{j}\right)\right]\right) \geq 0$ and $F\left(\left[u_{j}\left(w_{j}\right)\right]\right.$; $\left.\left[u_{i}\left(x_{i}\right), u_{j}\left(b_{j}\right)_{j \neq i}\right]\right) \geq 0$. If $u_{i}\left(z_{i}\right)>u_{i}\left(x_{i}\right)$ then $F\left(\left[u_{i}\left(z_{i}\right), u_{j}\left(a_{j}\right)_{j \neq i}\right] ;\left[u_{j}\left(y_{j}\right)\right]\right)>$ 0 so that $\left(z_{i}, a_{-i}\right) \succsim y$. Otherwise $u_{i}\left(x_{i}\right) \geq u_{i}\left(z_{i}\right)$ leads to $F\left(\left[u_{j}\left(w_{j}\right)\right]\right.$; $\left.\left[u_{i}\left(z_{i}\right), u_{j}\left(b_{j}\right)_{j \neq i}\right]\right) \geq 0$ so that $w \succsim\left(z_{i}, b_{-i}\right)$.

Sufficiency. Since $A C 1, A C 2$ and $A C 3$ hold, we know from part 4 of lemma 3 that $\succsim_{i}^{ \pm}$is a weak order. Since $X_{i}$ is countable, there is a realvalued function $u_{i}$ on $X_{i}$ such that, for all $x_{i}, y_{i} \in X_{i}$ :

$$
x_{i} \succsim_{i}^{ \pm} y_{i} \Leftrightarrow u_{i}\left(x_{i}\right) \geq u_{i}\left(y_{i}\right)
$$

Consider, for each $i \in N$, any real-valued function $u_{i}$ on $X_{i}$ satisfying (29) and define $F$ on $\left[\prod_{i=1}^{n} u_{i}\left(X_{i}\right)\right]^{2}$ letting, for all $x, y \in X$,

$$
F\left(\left[u_{i}\left(x_{i}\right)\right] ;\left[u_{i}\left(y_{i}\right)\right]\right)= \begin{cases}+\exp \left(\sum_{i=1}^{n}\left(u_{i}\left(x_{i}\right)-u_{i}\left(y_{i}\right)\right)\right) & \text { if } x \succsim y, \\ -\exp \left(\sum_{i=1}^{n}\left(u_{i}\left(y_{i}\right)-u_{i}\left(x_{i}\right)\right)\right) & \text { otherwise. }\end{cases}
$$

The well-definedness of $F$ follows from (9). The monotonicity properties of $F$ follow from (8) and its definition.

The impact of the reflexivity of $\succsim$ on $F$ is obvious.

It should be noted that a somewhat weaker form (using nondecreasingness and nonincreasingness) of theorem 2 was noted in Greco et al. (2002, Theorem 2.1) using our conditions $A C 1, A C 2$ and $A C 3$. 
The situation is slightly more complex with complete relations $\succsim$ if we insist on using a "skew symmetric" function $F$ (i.e., such that $F\left(\left[u_{i}\left(x_{i}\right)\right]\right.$; $\left.\left.\left[u_{i}\left(y_{i}\right)\right]\right)=-F\left(\left[u_{i}\left(y_{i}\right)\right] ;\left[u_{i}\left(x_{i}\right)\right]\right)\right)$. When $F$ is skew symmetric, the value " 0 " plays a special role. This leads to distinguish the increasing case from the nondecreasing one, as in proposition 5 with semi-orders and weak orders.

Theorem 3 (Skew symmetric representations on product sets) Let $\succsim$ be a binary relation on a countable set $X=\prod_{i=1}^{n} X_{i}$.

1. There is a numerical representation of type (27) in which $F$ is skew symmetric, nondecreasing in its first $n$ arguments and nonincreasing in its last $n$ arguments

iff

$\succsim$ is complete and satisfies $A C 1, A C 2$ and $A C 3$.

2. There is a numerical representation of type (27) in which $F$ is skew symmetric, increasing in its first $n$ arguments and decreasing in its last $n$ arguments

iff

$\succsim$ is complete and satisfies TAC1 and TAC2.

\section{ProOF}

Part 1. The necessity of completeness, $A C 1, A C 2$ and $A C 3$ is easily shown using the properties of $F$. We establish sufficiency. Consider, for each $i \in N$, any real-valued function $u_{i}$ on $X_{i}$ satisfying (29) and define $F$ on $\left[\prod_{i=1}^{n} u_{i}\left(X_{i}\right)\right]^{2}$ letting, for all $x, y \in X$,

$$
F\left(\left[u_{i}\left(x_{i}\right)\right] ;\left[u_{i}\left(y_{i}\right)\right]\right)=\left\{\begin{array}{cl}
+\exp \left(\sum_{i=1}^{n}\left(u_{i}\left(x_{i}\right)-u_{i}\left(y_{i}\right)\right)\right) & \text { if } x \succ y, \\
0 & \text { if } x \sim y, \\
-\exp \left(\sum_{i=1}^{n}\left(u_{i}\left(y_{i}\right)-u_{i}\left(x_{i}\right)\right)\right) & \text { otherwise }
\end{array}\right.
$$

The well-definedness of $F$ follows from (9). It is skew symmetric by construction since $\succsim$ is complete. Let us show that $F$ is nondecreasing in its first $n$ arguments. Suppose that $u_{i}\left(z_{i}\right)>u_{i}\left(x_{i}\right)$ so that $z_{i} \succ_{i}^{ \pm} x_{i}$. If $x \succ y$, we know, using (8), that $\left(z_{i}, x_{-i}\right) \succ y$ and the conclusion follows from the definition of $F$. If $x \sim y$, we have, using (8), $\left(z_{i}, x_{-i}\right) \succsim y$ and the conclusion follows from the definition of $F$. If $N$ ot $[x \succsim y]$ we have either $\left(z_{i}, x_{-i}\right) \succ y$, $\left(z_{i}, x_{-i}\right) \sim y$, or $\operatorname{Not}\left[\left(z_{i}, x_{-i}\right) \succsim y\right]$. In either case, the conclusion follows from the definition of $F$. The proof that $F$ is nonincreasing in its last $n$ argument is similar and is omitted.

Part 2. Necessity. The necessity of completeness is clear. Suppose that $\left(x_{i}, a_{-i}\right) \succsim y, y \succsim\left(z_{i}, a_{-i}\right),\left(z_{i}, b_{-i}\right) \succsim w$ and $\operatorname{Not}\left[\left(x_{i}, b_{-i}\right) \succsim w\right]$. Using the 
increasingness of $F$ in its first $n$ arguments, the last two conditions imply that $u_{i}\left(z_{i}\right)>u_{i}\left(x_{i}\right)$. But $\left(x_{i}, a_{-i}\right) \succsim y$ and $u_{i}\left(z_{i}\right)>u_{i}\left(x_{i}\right)$ imply $\left(z_{i}, a_{-i}\right) \succ y$, a contradiction. Hence the necessity of $T A C 1$. The necessity is $T A C 2$ is proved similarly.

Sufficiency. Since $\succsim$ is complete, we know that TAC1 and TAC2 imply $A C 1, A C 2$ and $A C 3$. Define $u_{i}$ and $F$ as in the proof of part 1 above. We have to show that $F$ is increasing. This results from the definition of $F$ and parts 2 and 6 of lemma 4 .

\subsection{Weak-orders}

In this section, we show how the preceding results particularize when it is supposed that $\succsim$ is a weak order. Since marginal traces are then confounded with marginal preferences, much simplification is expected.

Our first elementary result shows that the technique of proposition 6 applies to the classical numerical representation of weak orders.

\section{Proposition 7}

Let $\succsim$ be a binary relation on a countable set $X=\prod_{i=1}^{n} X_{i}$. There are realvalued functions $u_{i}$ on $X_{i}$ and a real-valued function $U$ on $\prod_{i=1}^{n} u_{i}\left(X_{i}\right)$ such that, for all $x, y \in X$,

$$
x \succsim y \Leftrightarrow U\left(u_{1}\left(x_{1}\right), \ldots, u_{n}\left(x_{n}\right)\right) \geq U\left(u_{1}\left(y_{1}\right), \ldots, u_{n}\left(y_{n}\right)\right) \geq 0,
$$

iff $\succsim$ is a weak order.

\section{PROOF}

Necessity is obvious. Since $\succsim$ is a weak order and $X$ is countable, there is a real-valued function $u$ on $X$ such that, for all $x, y \in X, x \succsim y \Leftrightarrow u(x) \geq u(y)$. Consider, for each $i \in N$, a real-valued function $u_{i}$ on $X_{i}$ satisfying (28) and define $U$ on $\prod_{i=1}^{n} u_{i}\left(X_{i}\right)$ letting, for all $x \in X$,

$$
U\left(\left[u_{i}\left(x_{i}\right)\right]\right)=u(x) .
$$

Using the reflexivity and transitivity of $\sim$ and $(9)$ it is easily shown that $U$ is well-defined.

Combining the results in lemmas 3,4 and 5 leads to the following.

\section{Proposition 8}

Let $\succsim$ be a weak order on a countable set $X=\prod_{i=1}^{n} X_{i}$. The function $U$ in (31) can be chosen to be: 
1. nondecreasing in each of its arguments

iff

$\succsim$ is weakly separable,

2. increasing in each of its arguments

iff

$\succsim$ is weakly independent.

\section{PROOF}

Part 1. Necessity of weak separability directly results from the nondecreasingness of $U$ in all its arguments and the reflexivity of $\succsim$. In order to prove sufficiency, we know from part 1 of lemma 5 that $A C 1, A C 2$ and $A C 3$ hold so that, using part 4 of lemma $3, \succsim_{i}^{ \pm}$is a weak order. Since $X_{i}$ is countable, there is a real-valued function $u_{i}$ on $X_{i}$ satisfying (29). Consider, for each $i \in N$, a real-valued function $u_{i}$ on $X_{i}$ satisfying (29) and define $U$ on $\prod_{i=1}^{n} u_{i}\left(X_{i}\right)$ as in in (32). The well-definedness of $U$ results from proposition 7 . The nondecreasingness of $U$ follows from (8) and its definition.

Part 2. Necessity of weak independence directly results from the increasingness of $U$ in all its arguments and the reflexivity of $\succsim$. Using functions $u_{i}$ and $U$ as in part 1, increasingness follows from (8) together with part 2 of lemma 5 and lemma 4.

Part 1 of proposition 8 generalizes a result obtained in Blackorby et al. (1978) in case $X \subseteq \mathbb{R}^{n}$ and was anticipated, in a different framework, in Greco, Matarazzo, and Słowiński (2001a). Part 2 is a well-known result (Krantz et al., 1971, theorem 7.1).

\subsection{Remarks}

The results in this section prompt a number of remarks.

1. Combining the results of theorems 1 and 2 shows, as announced, that all binary relations compatible with dominance, whether or not transitive and complete, have a nontrivial numerical representation. We therefore hope that our framework and results may serve to establish connections between the two traditions in decision analysis with multiple attributes mentioned in introduction. Using the idea of traces makes it possible to extend the traditional framework of conjoint measurement to analyze binary relations that may not be well-behaved. The need for studying such extensions was forcefully advocated in Bouyssou and Pirlot (2002a), Fishburn (1990, 1991a, 1991b), May (1954), Tversky (1969). 
Conversely the very intuitive but sometimes rather ad-hoc aggregation models based on the notion of dominance can be subjected to a standard axiomatic analysis in the framework of conjoint measurement.

2. The price to pay for such an extension of the scope of conjoint measurement is that our results, although constructive, are not well adapted to serve as a basis for assessment procedures. The general idea here is to use numerical representations as guidelines to understand the consequences of a limited number of cancellation conditions, without imposing any transitivity or completeness requirement on the preference relation and any structural assumptions on the set of objects. As already noticed in Bouyssou and Pirlot (2002a), such a poor framework happens to be surprisingly rich.

3. It should be clear that the numerical representations envisaged in this paper (see theorems 2 and 3) do not possess any remarkable uniqueness properties. Again, this is in line with our use of numerical representations as guidelines to investigate the consequences of some particular conditions on $\succsim$ and not as a direct basis to derive assessment procedures. We analyze the uniqueness properties of the representations in theorems 2 and 3 in appendix $\mathrm{C}$

4. Most of our results are technically simple. Their extension to the case in which $X$ is no more supposed to be countable, as shown in appendix B, do not raise any serious difficulty beyond the well-known one of guaranteeing that traces have a numerical representation. Therefore we refrained from spelling out the various possible extensions of our results beyond what we felt necessary for our purposes. Let us simply mention that we did not cover in this paper the case in which $A C 1$ and $A C 2$ hold but $A C 3$ is not imposed. The similarity of that case with that of interval orders (see Fishburn, 1970a, 1973b, 1985) should be clear at this point. Many of our results on product sets can easily be modified to cover that case using two real-valued functions $u_{i}$ and $v_{i}$ instead of one. We do not develop this point.

5. We restricted our attention in this paper to the analysis of conditions $A C 1_{i}, A C 2_{i}, A C 3_{i}, A C 4_{i}, T A C 1_{i}$ and $T A C 2_{i}$ when imposed for all $i \in$ $N$. As observed in Greco et al. (2002), this might be overly restrictive. It is not difficult however to study the, rather awkward, models that obtain when these conditions are only imposed on some, but not all attributes. 
Similarly, it is easy to generalize our conditions to subsets of attributes more general than a singleton. The study of the resulting models certainly deserves attention. In fact, when aggregating attributes, it might well happen that attributes interact in such a way that weak separability is violated. This would forbid the use of $A C 1$ or $A C 2$ as done here. Imposing these conditions on the groups of "strongly interacting" attributes might however lead to useful models. Such models would be in the spirit of the process of "building criteria" by sub-aggregation as described in e.g. Bouyssou (1990), Roy (1996).

\section{Discussion}

The main aim of this paper was to establish connections between the two separate traditions in decision analysis with multiple attributes mentioned in introduction. We believe that our framework based on the analysis of marginal traces does so. Although further research in this direction is obviously needed, our results give reasonable hope that it could be fruitful.

We conclude with some remarks and the indication of possible directions for future research.

1. The idea that the study of traces on attributes may offer insights on the structure of multi-attributed preferences also underlies the results in Bouyssou and Pirlot (2002a). Instead of studying traces on elements of $X_{i}$, we study traces on ordered pairs of elements of $X_{i}$ interpreted as a relation comparing "preference differences" defined from $\succsim$. More precisely, it is clear that the binary relation $\succsim_{i}^{*}$ on $X_{i}^{2}$ defined letting, for all $x_{i}, y_{i}, z_{i}, w_{i} \in X_{i}$,

$$
\begin{aligned}
& \left(x_{i}, y_{i}\right) \succsim_{i}^{*}\left(z_{i}, w_{i}\right) \text { iff } \\
& \quad\left[\text { for all } a_{-i}, b_{-i} \in X_{-i},\left(z_{i}, a_{-i}\right) \succsim\left(w_{i}, b_{-i}\right) \Rightarrow\left(x_{i}, a_{-i}\right) \succsim\left(y_{i}, b_{-i}\right)\right] .
\end{aligned}
$$

is always reflexive and transitive. This suggests a numerical representation of the type:

$$
x \succsim y \Leftrightarrow F\left(p_{1}\left(x_{1}, y_{1}\right), p_{2}\left(x_{2}, y_{2}\right), \ldots, p_{n}\left(x_{n}, y_{n}\right)\right) \geq 0,
$$

where $p_{i}$ are real-valued functions on $X_{i}^{2}$ and $F$ is a real-valued function on $\prod_{i=1}^{n} p_{i}\left(X_{i}^{2}\right)$, Imposing additional conditions on $p_{i}$ (e.g. their skew symmetry) and/or on $F$ (e.g. its oddness or nondecreasingness in all arguments) leads to a large variety of models that require the completeness of $\succsim_{i}^{*}$. 
As shown in Bouyssou and Pirlot (2002b), this family of models exploiting traces on "differences" is, in general, quite independent of the family of models exploiting traces on "levels" as studied here. This gives room for the study of models combining these two aspects, which is undertaken in Bouyssou and Pirlot (2002b). These hybrid models combining traces on "levels" and traces on "differences" are of the following type:

$$
x \succsim y \Leftrightarrow F\left(\phi_{1}\left(u_{1}\left(x_{1}\right), u_{1}\left(y_{1}\right)\right), \ldots, \phi_{n}\left(u_{n}\left(x_{n}\right), u_{n}\left(y_{n}\right)\right)\right) \geq 0,
$$

where $u_{i}$ is a real-valued function on $X_{i}$ and $\phi_{i}$ is a real-valued function on $u_{i}\left(X_{i}\right)^{2}, F$ is a real-valued function on $\prod_{i \in N} \phi_{i}\left(u_{i}\left(X_{i}\right), u\left(X_{i}\right)\right)$ and $\phi_{i}$ and $F$ may have additional properties (e.g., $\phi_{i}$ is skew symmetric and/or nondecreasing in its first argument and nonincreasing in its second arguments, $F$ is odd and/or nondecreasing in its arguments).

2. It has sometimes been claimed that rule-based preference modelling is more "flexible" than "functional" preference modelling e.g. Azibi and Vanderpooten (2002, p. 275). As far as "rules" are designed so as to obey dominance (which is the case in the above-mentioned paper), our results show that such claims are not founded. Although it is true that rule based preference modelling may offer some advantages (i.e. the possibility to "explain" in a language close to the natural language the preference relation linking two alternatives), it is clearly very closely related to models admitting numerical representations as studied here. In fact our function $F$, the precise functional form of which being unspecified, is a model that can be viewed as a "set of rules" indicating how to combine the various levels (the $u_{i}\left(x_{i}\right)$ 's) on each attribute. The close links between functional and rule-based models of preference have been already noted in Greco et al. (1999a, 1999b, 2001a, 2001b, 2002)

3. Our framework and results seem to be well-adapted to formalize the notion of "consistent family of criteria" as introduced in Roy and Bouyssou (1993), Roy (1996), Vincke (1992). Although this definition is somewhat more restrictive (requiring that combining "close levels", i.e. levels that are not identical but are related by $\sim_{i}$, should have a limited overall impact), it implies that any preference relation built on the basis of a consistent family of criteria is dominance compatible in the exact sense of definition 5. This shows that all preference relations obtained on the basis of a consistent family of criteria in the sense of Roy and Bouyssou (1993), Roy (1996), Vincke (1992) have a numerical representation of the type investigated in theorem 2. Therefore, subjecting our conditions to extensive empirical tests could offer a fresh 
view on the adequateness of common hypotheses adopted in decision analysis with several attributes.

Future research on the topics discussed in this paper could include:

- the extension of our results to the case of valued preference relations, an area in which the use of traces has already proved extremely useful (see Doignon et al., 1988; Monjardet, 1984; Roubens \& Vincke, 1985),

- the specialization of our results to the case of an homogeneous product set $\left(X_{i}=X_{j}, \forall i, j \in N\right)$, with applications to the field of decision under uncertainty,

- the use of the analogy between numerical representations used here and rule-based preference modelling to derive assessment procedures using the classical machinery of "rule induction" in Artificial Intelligence. This aspect has already been tackled in Greco et al. (1999b, 2001b, 2001a),

- the specialization of our results in order to study particular functional forms for $F$ such as min, max, Sugeno or Choquet integrals (see Grabisch, 1996; Marichal, 2000a, 2000b). Research in that direction has already started (see Bouyssou, Greco, Matarazzo, Pirlot, \& Słowiński, 2002). 


\section{Appendices}

\section{A Examples}

We first give three examples showing that, in the class of complete binary relations on $X, A C 1_{i}, A C 2_{i}$ and $A C 3_{i}$ are independent conditions. This will prove part 5 of lemma 3 . We leave to the reader the tedious, but easy, task of checking that $A C 1_{i}, A C 2_{i}$ and $A C 3_{i}$ are in fact completely independent in the class of complete binary relations.

Examples 1 to 3 have a common structure. In all of them $X=X_{1} \times$ $X_{2} \times X_{3}$ with $X_{1}=\{a, b, c\}, X_{2}=\{w, x, y\}$ and $X_{3}=\{q, r, s\}$. We abuse notation and write an element of $X$ as awq instead of $(a, w, q)$.

\section{Example 1}

Let $\succsim=X^{2}$ except that $N o t[a y q \succsim c w r], N o t[a y q \succsim c x r], N o t[a y s \succsim c w r]$, Not[ays $\succsim c x r], N o t[b w q \succsim c w r], N o t[b w q \succsim c x r], N o t[b y q \succsim c w r]$ and $\operatorname{Not}[b y q \succsim c x r]$.

It is not difficult to check that $\succsim$ is complete (it is in fact a weakly independent semi-order). A routine check shows that $A C k_{i}$ hold for all $k \in$ $\{1,2,3\}$ and $i \in\{1,2,3\}$ except that $A C 1_{1}$ fails. Indeed, we have $N o t\left[b \succsim_{1}^{+} a\right]$ (since $a w q \succsim c x r$ and $N o t[b w q \succsim c x r]$ ) and $N o t\left[a \succsim_{1}^{+} b\right]$ (since bys $\succsim c w r$ and $\operatorname{Not}[$ ays $\succsim c w r]$ ). It is not difficult to check that we have (using obvious notation for weak orders): $c \succ_{1}^{-}[a, b], x \succ_{2}^{+} w \succ_{2}^{+} y,[x, w] \succ_{2}^{-} y, r \succ_{3}^{+} s \succ_{3}^{+}$ $q$, and $r \succ_{3}^{-}[q, s]$. Hence we have an example of a complete binary relation satisfying $A C 2, A C 3$ and $A C 1_{i}$ on all attributes but $i=1$.

\section{Example 2}

Let $\succsim=X^{2}$ except that $\operatorname{Not}[c w r \succsim a y q], N o t[c w r \succsim a y s], N o t[c w r \succsim b w q]$, $\operatorname{Not}[c w r \succsim b y q], N o t[c x r \succsim a y q], N o t[c x r \succsim a y s], N o t[c x r \succsim b w q]$ and $\operatorname{Not}[\operatorname{cxr} \succsim b y q]$.

It is not difficult to check that $\succsim$ is is complete (it is in fact a weakly independent semi-order). A routine check shows that $A C k_{i}$ hold for all $k \in$ $\{1,2,3\}$ and $i \in\{1,2,3\}$ except that $A C 2_{1}$ fails. We have $N o t\left[a \succsim_{1}^{-} b\right]$ (since $c w r \succsim a w q$ and $N o t[c w r \succsim b w q]$ ) and $N o t\left[b \succsim_{1}^{-} a\right]$ (since $c w r \succsim b y s$ and $\operatorname{Not}[$ cwr $\succsim a y s]$ ). It is not difficult to check that we have (using obvious notation for weak orders): $[a, b] \succ_{1}^{+} c, y \succ_{2}^{+}[x, w], y \succ_{2}^{-} w \succ_{2}^{-} x,[q, s] \succ_{3}^{+} r$ and $q \succ_{3}^{-} s \succ_{3}^{-} r$.

Hence we have an example of a complete binary relation satisfying $A C 1$, $A C 3$ and $A C 2_{i}$ on all attributes but $i=1$.

\section{Example 3}

Let $\succsim=X^{2}$ except that $N o t[c y q \succsim a w q]$. 
It is not difficult to check that $\succsim$ is complete (it is in fact a weakly independent semi-order). A routine check shows that $A C k_{i}$ hold for all $k \in$ $\{1,2,3\}$ and $i \in\{1,2,3\}$ except that $A C 3_{3}$ fails. Indeed, we have: $[a, b] \succ_{1}^{+} c$, $a \succ_{1}^{-}[b, c],[w, x] \succ_{2}^{+} y, w \succ_{2}^{-}[x, y],[r, s] \succ_{3}^{+} q$ and $q \succ_{3}^{-}[r, s]$. This violates $A C 3_{3}$ since $r \succ_{3}^{+} q$ and $q \succ_{3}^{-} r$.

Hence we have an example of a complete binary relation satisfying $A C 1$, $A C 2$ and $A C 3_{i}$ on all attributes but $i=3$.

We leave to the reader the, easy, task of finding an example of a weakly independent semi-order satisfying $A C 1, A C 2$ and $A C 3$ but violating $A C 4$. The next two examples are related to lemma 4 . We first show that there are weakly independent semi-orders satisfying $A C 4$ that are not weak orders.

\section{Example 4}

Let $X=X_{1} \times X_{2}$ with $X_{1}=\left\{x_{1}, y_{1}, z_{1}\right\}$ and $X_{2}=\left\{x_{2}, y_{2}, z_{2}\right\}$. Consider the binary relation $\succsim$ identical to the complete order: $\left(x_{1}, x_{2}\right) \succ\left(x_{1}, y_{2}\right) \succ$ $\left(y_{1}, x_{2}\right) \succ\left(x_{1}, z_{2}\right) \succ\left(y_{1}, y_{2}\right) \succ\left(z_{1}, x_{2}\right) \succ\left(y_{1}, z_{2}\right) \succ\left(z_{1}, y_{2}\right) \succ\left(z_{1}, z_{2}\right)$, except that $\left(y_{1}, y_{2}\right) \sim\left(x_{1}, z_{2}\right)$ and $\left(z_{1}, x_{2}\right) \sim\left(y_{1}, y_{2}\right)$.

This relation is clearly complete. It is not transitive since $\left(z_{1}, x_{2}\right) \succsim$ $\left(y_{1}, y_{2}\right),\left(y_{1}, y_{2}\right) \succsim\left(x_{1}, z_{2}\right)$ but $\left(x_{1}, z_{2}\right) \succ\left(z_{1}, x_{2}\right)$.

It is easily checked that this relation is a semi-order having the preceding weak order for trace. This semi-order is independent. Its marginal relations are weak orders identical to its marginal traces. We have $x_{1} \succ_{1} y_{1} \succ_{1} z_{1}$ and $x_{2} \succ_{2} y_{2} \succ_{2} z_{2}$.

This relation has only a few pairs of alternatives linked by $\sim$. It is then easy to check that $A C 4$ holds using conditions (13) and (14). For instance, starting with $\left(y_{1}, y_{2}\right) \succsim\left(x_{1}, z_{2}\right)$ we should have $\left(x_{1}, y_{2}\right) \succ\left(x_{1}, z_{2}\right),\left(y_{1}, x_{2}\right) \succ$ $\left(x_{1}, z_{2}\right)$ and $\left(y_{1}, y_{2}\right) \succ\left(y_{1}, z_{2}\right)$, because $x_{1} \succ_{1}^{ \pm} y_{1}$ and $x_{2} \succ_{2}^{ \pm} y_{2}$. This is indeed the case.

Hence we have an example of a nontransitive weakly independent semiorder satisfying $A C 4$.

The final example shows that for complete relations, TAC2 may hold without $T A C 1$. An example of a complete relation verifying $T A C 1$ but not $T A C 2$ is easily built using a similar principle.

\section{Example 5}

Let $X=X_{1} \times X_{2}$ with $X_{1}=\mathbb{R} \times\{0 ; 2\}$ and $X_{2}=\mathbb{R}$.

Define $\succsim$ letting:

$$
\left(\left(a_{1}, b_{1}\right), x_{2}\right) \succsim\left(\left(c_{1}, d_{1}\right), y_{2}\right) \Leftrightarrow a_{1}+x_{2}>c_{1}+y_{2} \text { or }\left\{\begin{array}{l}
a_{1}+x_{2}=c_{1}+y_{2} \\
\text { and } \\
a_{1}+b_{1} \geq c_{1}
\end{array}\right.
$$


It is easy to check that $\succsim$ is complete.

On the second attribute, it is clear that $x_{2} \succsim_{2}^{+} y_{2} \Leftrightarrow x_{2} \succsim_{2}^{-} y_{2} \Leftrightarrow x_{2} \geq y_{2}$.

Suppose that $a_{1} \geq c_{1}$. Then, we clearly have $w \succsim\left(\left(a_{1}, b_{1}\right), y_{2}\right) \Rightarrow w \succsim$ $\left(\left(c_{1}, d_{1}\right), y_{2}\right)$, for all $b_{1}, d_{1} \in\{0 ; 2\}$.

As soon as $c_{1}>a_{1}$, it is clearly possible to have $w \succsim\left(\left(a_{1}, b_{1}\right), y_{2}\right)$ and $N o t\left[w \succsim\left(\left(c_{1}, d_{1}\right), y_{2}\right)\right]$. Therefore $\left(a_{1}, b_{1}\right) \succsim_{1}^{-}\left(c_{1}, d_{1}\right) \Leftrightarrow a_{1} \geq c_{1}$.

If $a_{1}>c_{1}$, it is clear that $\left(\left(c_{1}, d_{1}\right), y_{2}\right) \succsim z \Rightarrow\left(\left(a_{1}, b_{1}\right), y_{2}\right) \succsim z$.

If $a_{1}=c_{1}$, we have $\left(\left(c_{1}, 0\right), y_{2}\right) \succsim z \Rightarrow\left(\left(a_{1}, 0\right), y_{2}\right) \succsim z,\left(\left(c_{1}, 2\right), y_{2}\right) \succsim z$ $\Rightarrow\left(\left(a_{1}, 2\right), y_{2}\right) \succsim z$ and $\left(\left(c_{1}, 2\right), y_{2}\right) \succsim z \Rightarrow\left(\left(a_{1}, 0\right), y_{2}\right) \succsim z$. However we may have $\left(\left(c_{1}, 2\right), y_{2}\right) \succsim z$ and $\operatorname{Not}\left[\left(\left(a_{1}, 0\right), y_{2}\right) \succsim z\right]$. Therefore, we have

$$
\left(a_{1}, b_{1}\right) \succsim_{1}^{+}\left(c_{1}, d_{1}\right) \Leftrightarrow\left\{\begin{array}{l}
a_{1}>c_{1} \text { or } \\
a_{1}=c_{1} \text { and } b_{1} \geq d_{1} .
\end{array}\right.
$$

A simple check shows that $\succsim$ is strictly responsive to $\succsim_{2}^{+}, \succsim_{2}^{-}$and $\succsim_{1}^{-}$. This not so for $\succsim_{1}^{+}$. In fact, we have, $((10,0), 10) \sim((8,2), 12)$ and $((10,2), 10) \sim$ $((8,2), 12)$, while $(10,2) \succ_{1}^{+}(10,0)$ (because $((10,2), 10) \succsim((11,0), 9)$ and $\operatorname{Not}[((10,0), 10) \succsim((11,0), 9)])$.

Hence we have an example of a complete relation satisfying $T A C 2$ and $T A C 1_{2}$ but violating $T A C 1_{1}$.

\section{B Numerical representations: the general case}

Let $E$ be an equivalence on a set $A$. We say that $A$ satisfies the low cardinality condition w.r.t. $E(L C C[A / E])$ if there is a one-to-one correspondence between $A / E$ and some subset of $\mathbb{R}$. As soon as $E$ is an equivalence relation, condition $L C C[A / E]$ is clearly necessary and sufficient for the existence of a real-valued function $f$ on $A$ such that, for all $a, b \in A$ :

$$
a E b \Leftrightarrow f(a)=f(b) .
$$

Condition $L C C[A / E]$ is very mild and is clearly satisfied as soon as $A$ is some subset of $\mathbb{R}^{k}$.

Let $S$ be a binary relation on a set $A$ and let $B \subseteq A$. Following e.g. Krantz et al. (1971, Chapter 2), we say that $B$ is dense in $A$ for $S$ if, for all $a, b \in A,[a S b$ and $N o t[b S a]] \Rightarrow[a S c$ and $c S b$, for some $c \in B]$. The existence of a finite or countably infinite set $B$ dense in $A$ for $S$ is a necessary condition for the existence of a real-valued function $f$ on $A$ such that, for all $a, b \in A$, $a S b \Leftrightarrow f(a) \geq f(b)$. Together with the fact that $S$ is a weak order on $A$, it is also sufficient for the existence of such a representation (see Fishburn (1970b) or Krantz et al. (1971)). 
We say that a binary relation $\succsim$ on $A$ satisfies condition $O D$ (Order Density) if there is a countable subset $B \subseteq A$ that is dense in $A$ for $\succsim$. We say that $\succsim$ on $A$ satisfies condition $O D^{ \pm}$if there is a countable subset $B \subseteq A$ that is dense in $A$ for $\succsim^{ \pm}$. Clearly, if $\succsim$ is a weak order on A, $O D$ and $O D^{ \pm}$ are equivalent since in this case $\succsim=\succsim^{ \pm}$. The formulation of $O D^{ \pm}$in terms of $\succsim$ is cumbersome and apparently uninformative; for a thorough analysis of various conditions guaranteeing that traces have a numerical representation, we refer to Beja and Gilboa (1992), Candeal, Induráin, and Zudaire (2002), Doignon, Ducamp, and Falmagne (1984), Fishburn (1985), Nakamura (2002), Narens (1994), Oloriz, Candeal, and Induráin (1998).

Let $\succsim$ and $\succsim^{\prime}$ be two weak orders on $A$. We say that $\succsim^{\prime}$ refines $\succsim$ if, for all $a, b \in A, a \succsim^{\prime} b \Rightarrow a \succsim b$. It is easy to see that if $\succsim^{\prime}$ refines $\succsim$ and $\succsim^{\prime}$ satisfies $O D$ then $\succsim$ satisfies $O D$.

When $\succsim$ is a binary relation on a product set $X=X_{1} \times X_{2} \times \cdots \times X_{n}$ we say that it satisfies condition $O D_{i}^{ \pm}$if there is a countable set $B$ that is dense in $X_{i}$ for $\succsim_{i}^{ \pm}$.

Using these conditions, we first tackle the case of trivial representations on sets without structure. For the sake of completeness, we spell out the following:

\section{Proposition 9 (Generalization of propositions 4 and 5)}

When removing the restriction that $A$ is finite or countably infinite,

1. Proposition 4 holds iff $\succsim$ satisfies $L C C\left[A / \sim^{ \pm}\right]$.

2. Parts 1 and 2 of proposition 5 hold iff $\succsim$ satisfies $O D^{ \pm}$.

3. Part 3 of proposition 5 holds iff $\succsim$ satisfies $O D$.

\section{PROOF}

Part 1 is obvious. The sufficiency of $O D^{ \pm}$(resp. $O D$ ) for part 2 (resp. part 3) is clear.

Let us prove the necessity of $O D^{ \pm}$. Suppose that $a \succ^{ \pm} b$. By definition, there is a $c \in A$ such that either $[a \succsim c$ and $\operatorname{Not}[b \succsim c]]$ or $[c \succsim b$ and $\operatorname{Not}[c \succsim$ $a]$. In the first case, we have: $\mathcal{F}(u(a), u(c)) \geq 0$ and $\mathcal{F}(u(b), u(c))<0$. In the second case, we obtain: $\mathcal{F}(u(c), u(b)) \geq 0$ and $\mathcal{F}(u(c), u(a))<0$. Therefore, when $\mathcal{F}$ is nondecreasing in its first argument and nonincreasing in its second argument, representation (22) implies:

$$
a \succ^{ \pm} b \Rightarrow u(a)>u(b) .
$$

The necessity of $O D^{ \pm}$follows since the weak order induced on $A$ by $u$ refines $\succsim^{ \pm}$. The necessity of $O D$ for part 3 is proven in a similar way. 
The generalization of proposition 6 is done along the same lines. When $X$ is no longer supposed to be countable, it is necessary and sufficient to require that condition $L C C\left[X_{i} / \sim_{i}^{ \pm}\right]$holds for all $i \in N$. This is not worth spelling out in detail (note however that it is not difficult to show that condition $L C C\left[X / \sim^{ \pm}\right]$implies that condition $L C C\left[X_{i} / \sim_{i}^{ \pm}\right]$holds for all $\left.i \in N\right)$.

Similarly to what has been done in the proof of proposition 9 , it is not difficult to show that when $\succsim$ has a numerical representation of type (27) with $F$ being nondecreasing (resp. nonincreasing) in its first (resp. last) $n$ arguments then, for all $i \in N$ and all $x_{i}, y_{i} \in X_{i}$ :

$$
x_{i} \succ_{i}^{ \pm} y_{i} \Rightarrow u_{i}\left(x_{i}\right)>u_{i}\left(y_{i}\right)
$$

The necessity of condition $O D_{i}^{ \pm}$for all $i \in N$ therefore follows. We have:

\section{Proposition 10 (Generalization of theorems 2 and 3)}

When removing the condition that $X$ is finite or countably infinite, Theorems 2 and 3 hold iff $\succsim$ satisfies $O D_{i}^{ \pm}$for all $i \in N$.

We leave to the interested reader the construction of examples showing that $O D_{i}^{ \pm}$may hold for all $i \in N \backslash\{j\}$ while $O D_{j}^{ \pm}$fails.

In order to generalize proposition 7 , it must clearly be supposed that $\succsim$ satisfies $O D$. Since we do not suppose here substituability as in Krantz et al. (1971, Theorem 7.1), we also have to suppose $L C C\left[X_{i} / \sim_{i}^{ \pm}\right]$holds for all $i \in N$. The following example shows that $L C C\left[X_{i} / \sim_{i}^{ \pm}\right]$is independent from $O D$.

\section{Example 6 (OD and $\mathrm{LCC}\left[\mathrm{X}_{\boldsymbol{i}} / \sim_{\boldsymbol{i}}^{ \pm}\right]$)}

Let $X=X_{1} \times X_{2}$ with $X_{1}=X_{2}=2^{\mathbb{R}}$, the set of all subsets of $\mathbb{R}$. Define $\succsim$ on $X$ letting, for all $A, B, C, D \in 2^{\mathbb{R}},(A, B) \succsim(C, D) \Leftrightarrow f(A, B) \geq f(C, D)$, where $f$ is a real-valued function on $\left[2^{\mathbb{R}}\right]^{2}$ such that $f(A, B)=1 \Leftrightarrow B \subseteq A$ and $f(A, B)=0$ otherwise.

By construction, $\succsim$ is a weak order satisfying $O D$. However, as soon as $A \neq B$, it is clear that $\operatorname{Not}\left[A \sim_{1}^{ \pm} B\right]$ and $\operatorname{Not}\left[A \sim_{2}^{ \pm} B\right]$. Hence, $L C C\left[X_{i} / \sim_{i}^{ \pm}\right]$ is violated.

The generalization of part 2 of proposition 8 is classical (Krantz et al., 1971, theorem 7.1). Since for weak orders, marginal preferences and marginal traces coincide, it suffices to impose that the weak order $\succsim$ has a numerical representation, i.e., that $O D$ holds. The generalization of part 1 is somewhat trickier since there are weakly separable weak orders that have a numerical representation while their marginal traces do not (see Fishburn, 1973a, Theorem A(ii)). Hence it must also be added that condition $O D_{i}^{ \pm}$holds for all $i \in N$. We summarize our observations below. 


\section{Proposition 11 (Generalization of propositions 7 and 8)}

When removing the condition that $X$ is finite or countably infinite,

1. Proposition 7 holds iff $\succsim$ satisfies $O D$ and $L C C\left[X_{i} / \sim_{i}^{ \pm}\right]$, for all $i \in N$.

2. Part 1 of proposition 8 holds iff $\succsim$ satisfies $O D$ and $O D_{i}^{ \pm}$, for all $i \in N$.

3. Part 2 of proposition 8 holds iff $\succsim$ satisfies $O D$.

\section{Uniqueness}

Let us first envisage the case of theorem 2 (without reflexivity). The numerical representation is such that:

$$
x \succsim y \Leftrightarrow F\left(\left[u_{i}\left(x_{i}\right)\right] ;\left[u_{i}\left(y_{i}\right)\right]\right) \geq 0,
$$

with $F$ increasing in its first $n$ arguments and decreasing in its last $n$ arguments. The proof of theorem 2 shows that it is always possible to build a numerical representation such that:

$$
x_{i} \succsim_{i}^{ \pm} y_{i} \Leftrightarrow u_{i}\left(x_{i}\right) \geq u_{i}\left(y_{i}\right) .
$$

This not compulsory however. Let us show that any function $u_{i}$ such that:

$$
x_{i} \succ_{i}^{ \pm} y_{i} \Rightarrow u_{i}\left(x_{i}\right)>u_{i}\left(y_{i}\right),
$$

can be used in a representation of type (38).

The necessity of (40) is clear since $x_{i} \succ_{i}^{ \pm} y_{i}$ implies either $x_{i} \succ_{i}^{+} y_{i}$ or $x_{i} \succ_{i}^{-} y_{i}$. In the first case, we know that $\left(x_{i}, a_{-i}\right) \succsim z$ and $\operatorname{Not}\left[\left(y_{i}, a_{-i}\right) \succsim z\right]$, for some $z \in X$ and some $a_{-i} \in X_{-i}$. In the second case, we obtain $w \succsim$ $\left(y_{i}, b_{-i}\right)$ and $\operatorname{Not}\left[w \succsim\left(x_{i}, b_{-i}\right)\right]$, for some $w \in X$ and some $b_{-i} \in X_{-i}$. Using the increasingness of $F$, either case implies $u_{i}\left(x_{i}\right)>u_{i}\left(y_{i}\right)$.

Conversely, it is clear that if $u_{i}$ satisfies (40) then

$$
u_{i}\left(x_{i}\right)=u_{i}\left(y_{i}\right) \Rightarrow x_{i} \sim_{i}^{ \pm} y_{i},
$$

so that defining $F$, as in the proof of theorem 2, letting:

$$
F\left(\left[u_{i}\left(x_{i}\right)\right] ;\left[u_{i}\left(y_{i}\right)\right]= \begin{cases}+\exp \left(\sum_{i=1}^{n}\left(u_{i}\left(x_{i}\right)-u_{i}\left(y_{i}\right)\right)\right) & \text { if } x \succsim y \\ -\exp \left(\sum_{i=1}^{n}\left(u_{i}\left(y_{i}\right)-u_{i}\left(x_{i}\right)\right)\right) & \text { otherwise. }\end{cases}\right.
$$

leads to a well-defined function being increasing in its first $n$ arguments and decreasing in its last $n$ arguments. 
It should be noted that any real-valued functions on $\mathbb{R}^{2 n}$ (restricted to $\left.\left[\prod_{i=1}^{n} u_{i}\left(X_{i}\right)\right]^{2}\right)$ increasing in its first $n$ arguments and decreasing in its last $n$ arguments may be used instead of the particular one chosen in (42). It is furthermore clear that only such functions may be used.

We have therefore described the set of all possible numerical representations of type (38).

Let us now consider the case of the skew symmetric representations of theorem 3. When it is only required that $F$ is nondecreasing in its first $n$ arguments and nonincreasing in its last $n$ arguments, it is not difficult to see that the above reasoning applies. The combination of any real-valued function satisfying (40) with any any real-valued functions on $\mathbb{R}^{2 n}$ (restricted to $\left.\left[\prod_{i=1}^{n} u_{i}\left(X_{i}\right)\right]^{2}\right)$ nondecreasing in its first $n$ arguments and nonincreasing in its last $n$ gives an acceptable numerical representation.

The situation is slightly more complex in the skew symmetric case with $F$ increasing in its first $n$ arguments and decreasing in its last $n$ arguments. In that case, any function satisfying (40) will not do any more. To see why this happens, suppose that $x_{i} \sim_{i}^{ \pm} z_{i}$ and $u_{i}\left(x_{i}\right)>u_{i}\left(z_{i}\right)$. This is acceptable as long as it never happens that $\left(x_{i}, a_{-i}\right) \sim w$ because the increasingness of $F$ would then imply $\left(z_{i}, a_{-i}\right) \succ w$, violating (9). However, it is clear that the presence of $\sim$ is the only additional constraint preventing from choosing different values of $u_{i}$ for elements linked by $\sim_{i}^{ \pm}$. Therefore, in the increasing/decreasing skew symmetric model any $u_{i}$ such that:

$$
\left.\begin{array}{c}
x_{i} \succ_{i}^{ \pm} y_{i} \Rightarrow u_{i}\left(x_{i}\right)>u_{i}\left(y_{i}\right) \\
\text { and } \\
x_{i} \sim_{i}^{ \pm} y_{i} \\
\text { and } \\
\left(x_{i}, a_{-i}\right) \sim w \text { for some } a_{-i} \in X_{-i} \text { and some } w \in X
\end{array}\right\} \Rightarrow u_{i}\left(x_{i}\right)=u_{i}\left(y_{i}\right),
$$

is acceptable and can be combined with any real-valued function on $\mathbb{R}^{2 n}$ (restricted to $\left.\left[\prod_{i=1}^{n} u_{i}\left(X_{i}\right)\right]^{2}\right)$ increasing in its first $n$ arguments and decreasing in its last $n$ arguments.

\section{References}

Aleskerov, F., \& Monjardet, B. (2002). Utility maximization, choice and preference. Heidelberg: Springer-Verlag.

Azibi, R., \& Vanderpooten, D. (2002). Construction of rule-based assignment models. European Journal of Operational Research, 138, 274-293. 
Beja, A., \& Gilboa, I. (1992). Numerical representations of imperfectly ordered preferences (a unified geometric exposition). Journal of Mathematical Psychology, 36, 426-449.

Belton, V., \& Stewart, T. (2001). Multiple criteria decision analysis: An integrated approach. Dordrecht: Kluwer.

Blackorby, C., Primont, D., \& Russell, R. (1978). Duality, separability, and functional structure: Theory and economic applications. New-York: North-Holland.

Bouyssou, D. (1990). Building criteria: A prerequisite for MCDA. In C. Bana e Costa (Ed.), Readings in multiple criteria decision aid (pp. 58-80). Springer-Verlag.

Bouyssou, D., Greco, S., Matarazzo, B., Pirlot, M., \& Słowiński, R. (2002, July). Characterization of 'max', 'min' and 'order statistics' multicriteria aggregation functions. (Communication to IFORS'2002, 8 - 12 July, 2002, Edinburgh, U.K.)

Bouyssou, D., Marchant, T., Pirlot, M., Perny, P., Tsoukiàs, A., \& Vincke, P. (2000). Evaluation and decision models: a critical perspective. Dordrecht: Kluwer.

Bouyssou, D., \& Pirlot, M. (1999). Conjoint measurement without additivity and transitivity. In N. Meskens \& M. Roubens (Eds.), Advances in decision analysis (pp. 13-29). Dordrecht: Kluwer.

Bouyssou, D., \& Pirlot, M. (2002a). Non transitive decomposable conjoint measurement. forthcoming in Journal of Mathematical Psychology.

Bouyssou, D., \& Pirlot, M. (2002b). 'Additive difference' models without additivity and subtractivity. (Working Paper)

Bouyssou, D., Pirlot, M., \& Vincke, Ph. (1997). A general model of preference aggregation. In M.H. Karwan, J. Spronk, \& J. Wallenius (Eds.), Essays in decision making (pp. 120-134). Berlin: Springer Verlag.

Candeal, J., Induráin, E., \& Zudaire, M. (2002). Numerical representability of semiorders. Mathematical Social Sciences, 43, 61-77.

Debreu, G. (1960). Topological methods in cardinal utility theory. In S. K. K.J. Arrow \& P. Suppes (Eds.), Mathematical methods in the social sciences (pp. 16-26). Stanford: Stanford University Press. 
Doignon, J.-P., Ducamp, A., \& Falmagne, J.-C. (1984). On realizable biorders and the biorder dimension of a relation. Journal of Mathematical Psychology, 28, 73-109.

Doignon, J.-P., Monjardet, B., Roubens, M., \& Vincke, Ph. (1988). Biorder families, valued relations and preference modelling. Journal of Mathematical Psychology, 30, 435-480.

Ebert, U. (1985). Non-transitive representations of transitive orderings. Economic Letters, 18, 109-112.

Färe, R., \& Primont, D. (1981). Separability vs. weak separability: A further result. Journal of Economic Theory, 25, 455-460.

Fishburn, P.C. (1970a). Intransitive indifference in preference theory: A survey. Operations Research, 18(2), 207-228.

Fishburn, P.C. (1970b). Utility theory for decision-making. New-York: Wiley.

Fishburn, P.C. (1973a). Binary choice probabilities: On the varieties of stochastic transitivity. Journal of Mathematical Psychology, 10, 327352 .

Fishburn, P.C. (1973b). Interval representations for interval orders and semiorders. Journal of Mathematical Psychology, 10, 91-105.

Fishburn, P.C. (1985). Interval orders and intervals graphs. New-York: Wiley.

Fishburn, P.C. (1990). Additive non-transitive preferences. Economic Letters, 34, 317-321.

Fishburn, P.C. (1991a). Nontransitive preferences in decision theory. Journal of Risk and Uncertainty, 4, 113-134.

Fishburn, P.C. (1991b). Nontransitive additive conjoint measurement. Journal of Mathematical Psychology, 35, 1-40.

French, S. (1993). Decision theory - an introduction to the mathematics of rationality. London: Ellis Horwood.

Goicoechea, A., Hansen, D., \& Duckstein, L. (1982). Multiobjective decision analysis with engineering and business applications. New-York: Wiley.

Goldstein, W.M. (1991). Decomposable threshold models. Journal of Mathematical Psychology, 35, 64-79. 
Grabisch, M. (1996). The application of fuzzy integrals to multicriteria decision making. European Journal of Operational Research, 89, 445456.

Greco, S., Matarazzo, B., \& Słowiński, R. (1999a). Rough approximation of a preference relation by dominance relations. European Journal of Operational Research, 117, 63-83.

Greco, S., Matarazzo, B., \& Słowiński, R. (1999b). The use of rough sets and fuzzy sets in MCDM. In T. Gal, T. Hanne, \& T. Stewart (Eds.), Multicriteria decision making, Advances in MCDM models, algorithms, theory and applications (pp. 14.1-14.59). Dordrecht: Kluwer.

Greco, S., Matarazzo, B., \& Słowiński, R. (2001a). Conjoint measurement and rough set approach for multicriteria sorting problems in presence of ordinal criteria. In A. Colorni, M. Paruccini, \& B. Roy (Eds.), AMCD-A, aide mulcritère à la décision/multiple criteria decision aid (pp. 117-144). European Commission, Joint Research Centre.

Greco, S., Matarazzo, B., \& Słowiński, R. (2001b). Rough sets theory for multicriteria decision analysis. European Journal of Operational Research, 129, 1-7.

Greco, S., Matarazzo, B., \& Słowiński, R. (2002). Preference representation by means of conjoint measurement and decision rule model. In D. Bouyssou, E. Jacquet-Lagrèze, P. Perny, D. Vanderpooten, \& Ph. Vincke (Eds.), Aiding decisions with multiple criteria: Essays in honour of Bernard Roy (pp. 263-313). Dordrecht: Kluwer.

Keeney, R.L., \& Raiffa, H. (1976). Decisions with multiple objectives: Preferences and value tradeoffs. New-York: Wiley.

Krantz, D.H., Luce, R.D., Suppes, P., \& Tversky, A. (1971). Foundations of measurement, vol. 1: Additive and polynomial representations. NewYork: Academic Press.

Laslier, J.-F. (1997). Tournament solutions and majority voting. Heidelberg: Springer-Verlag.

Luce, R.D. (1956). Semi-orders and a theory of utility discrimination. Econometrica, 24, 178-191.

Luce, R.D., \& Tukey, J.W. (1964). Simultaneous conjoint measurement: a new type of fundamental measurement. Journal of Mathematical Psychology, 1, 1-27. 
Mak, K.-T. (1984). Notes on separable preferences. Journal of Economic Theory, 33, 309-321.

Marichal, J.-L. (2000a). On Sugeno integrals as an aggregation function. Fuzzy Sets and Systems, 114, 347-365.

Marichal, J.-L. (2000b). An axiomatic approach of the discrete Choquet integral as a tool to aggregate interacting criteria. IEEE Transactions on Fuzzy Systems, 8, 800-807.

May, K.O. (1954). Intransitivity, utility and the aggregation of preference patterns. Econometrica, 22, 1-13.

Monjardet, B. (1978). Axiomatiques et propriétés des quasi-ordres. Mathématiques et Sciences Humaines, 63, 51-82.

Monjardet, B. (1984). Probabilistic consistency, homogeneous families of relations and linear $\Lambda$-relations. In E. Degreef \& J. van Buggenhaut (Eds.), Trends in mathematical psychology (pp. 271-281). Amsterdam: North-Holland.

Nakamura, Y. (2002). Real interval representations. Journal of Mathematical Psychology, 46, 140-177.

Narens, L. (1994). The measurement theory of dense threshold structures. Journal of Mathematical Psychology, 38, 301-321.

Oloriz, E., Candeal, J., \& Induráin, E. (1998). Representability of interval orders. Journal of Economic Theory, 78, 219-227.

Pirlot, M., \& Vincke, Ph. (1997). Semiorders. Properties, representations, applications. Dordrecht: Kluwer.

Pomerol, J.-C., \& Barba-Romero, S. (2000). Multicriterion decision in management, principles and practice. Dordrecht: Kluwer.

Raiffa, H. (1968, December). Preference for multi-attributed alternatives. (RAND Memorandum, RM-5868-DOT/RC)

Roberts, F.S. (1979). Measurement theory with applications to decision making, utility and the social sciences. Reading: Addison-Wesley.

Roubens, M., \& Vincke, Ph. (1985). Preference modelling. Berlin: Springer Verlag. 
Roy, B. (1971). Problems and methods with multiple objective functions. Mathematical Programming, 1, 239-266.

Roy, B. (1991). The outranking approach and the foundations of ELECTRE methods. Theory and Decision, 31, 49-73.

Roy, B. (1996). Multicriteria methodology for decision aiding. Dordrecht: Kluwer. (Original version in French "Méthodologie multicritère d'aide à la décision", Economica, Paris, 1985)

Roy, B., \& Bouyssou, D. (1993). Aide multicritère à la décision : Méthodes et cas. Paris: Economica.

Scott, D. (1964). Measurement structures and linear inequalities. Journal of Mathematical Psychology, 1, 233-247.

Scott, D., \& Suppes, P. (1958). Foundational aspects of theories of measurement. Journal of Symbolic Logic, 23, 113-128.

Segal, U., \& Sobel, J. (2001). Min, Max and Sum. (Working Paper)

Sen, A.K. (1986). Social choice theory. In K.J. Arrow \& M.D. Intriligator (Eds.), Handbook of mathematical economics (Vol. 3, pp. 1073-1181). Amsterdam: North-Holland.

Steuer, R. (1986). Multiple criteria optimisation: Theory, computation, and application. New York: Wiley.

Tversky, A. (1969). Intransitivity of preferences. Psychological Review, 76, $31-48$.

Vincke, Ph. (1992). Multi-criteria decision aid. New York: Wiley. (Original version in French "L'Aide Multicritère à la Décision", Éditions de l'Université de Bruxelles-Éditions Ellipses, Brussels, 1989)

Wakker, P.P. (1989). Additive representations of preferences: A new foundation of decision analysis. Dordrecht: Kluwer.

Winterfeldt, D. von, \& Edwards, W. (1986). Decision analysis and behavioral research. Cambridge: Cambridge University Press.

Zeleny, M. (1982). Multiple criteria decision making. Mac Graw Hill. 